IGC- $-08 / 11-1$

\title{
Gauge invariant cosmological perturbation equations with corrections from loop quantum gravity
}

\author{
Martin Bojowald \\ Institute for Gravitation and the Cosmos, The Pennsylvania State University, \\ 104 Davey Lab, University Park, PA 16802, USA \\ Golam Mortuza Hossain \\ Department of Mathematics and Statistics, University of New Brunswick, \\ Fredericton, NB E3B 5A3, Canada \\ Mikhail Kagan \\ Department of Science and Engineering, The Pennsylvania State University, Abington \\ 1600 Woodland Road, Abington, PA 19116, USA \\ and \\ Institute for Gravitation and the Cosmos, The Pennsylvania State University, \\ 104 Davey Lab, University Park, PA 16802, USA \\ S. Shankaranarayanan \\ Institute of Cosmology and Gravitation, University of Portsmouth, Mercantile House, \\ Portsmouth P01 2EG, U.K.
}

\begin{abstract}
A consistent implementation of quantum gravity is expected to change the familiar notions of space, time and the propagation of matter in drastic ways. This will have consequences on very small scales, but also gives rise to correction terms in evolution equations of modes relevant for observations. In particular, the evolution of inhomogeneities in the very early universe should be affected. In this paper consistent evolution equations for gauge-invariant perturbations in the presence of inverse triad corrections of loop quantum gravity are derived. Some immediate effects are pointed out, for instance concerning conservation of power on large scales and non-adiabaticity. It is also emphasized that several critical corrections can only be seen to arise in a fully consistent treatment where the gauge freedom of canonical gravity is not fixed before implementing quantum corrections. In particular, metric modes must be allowed to be inhomogeneous: it is not consistent to assume only matter inhomogeneities on a quantum corrected homogeneous background geometry. In this way, stringent consistency conditions arise for possible quantization ambiguities which will eventually be further constrained observationally.
\end{abstract}




\section{Introduction}

General relativity describes the structure and dynamics of space-time by Einstein's equation for the space-time metric, which gives rise to a wide range of phenomena in cosmology and astrophysics. In several regimes, especially at high densities, one expects quantum gravity to be crucial and to provide important correction terms to the classical equations. By now, canonical quantum gravity in the loop approach has progressed to the extent that perturbative calculations for the behavior of inhomogeneities around Friedmann-Robertson-Walker space-times can be performed at an effective level. This provides cosmological applications, but by addressing the anomaly problem it also sheds light on fundamental aspects such as quantum space-time structure.

The classical equations of motion constitute an overdetermined set, whose consistency is ensured by general covariance. When attempting to include correction terms in these equations as they may be suggested by quantum gravity, the consistency conditions must be respected. Only an anomaly-free quantization, where consistency resulting from general covariance is preserved, can lead to quantum equations of motion with the correct number and behavior of degrees of freedom.

This issue becomes pressing already at the level of linearized equations as they are used in cosmology of the early universe. Once inhomogeneities are included as perturbations around an expanding Friedmann-Robertson-Walker space-time, there are more equations than unknowns which requires consistent forms of all terms in the equations. Without inhomogeneities, consistency is automatically satisfied: there is a single constraint which is always preserved by the evolution it generates. Quantum corrections to homogeneous models can therefore be implemented easily as it has been done often to suggest diverse effects and scenarios [1. However, just putting quantum corrected solutions for a homogeneous background into classical perturbation equations in general results in inconsistent equations: the corrected background equations can no longer be compatible with all the terms in the perturbation equations. Thus, a direct treatment of inhomogeneities and the corrections they acquire in a quantum theory of gravity is required.

Perturbations around homogeneous models then test their robustness, demonstrate whether a particular form of quantum corrections can be realized in a generally covariant way, and provide consistent sets of equations of motion whose solutions can be analyzed for the phenomenology and potentially observable effects they imply. From the perspective of canonical quantum gravity, the consistency issue of effective equations has been described in [2], and it has been demonstrated that there is a correction expected from loop quantum gravity [3, 4, 5] which non-trivially changes the classical equations in a consistent way at a perturbative level. (This correction results from inverse triad operators in Hamiltonians [6, 7]. Other expected corrections from loop quantum gravity, such as holonomy corrections, have not yet resulted in consistent equations outside the reduced setting of homogeneous models.) In this paper, we derive the corresponding gauge invariant perturbations and the equations of motion they satisfy.

Earlier work [8, 9] had already led to quantum corrections to Einstein's equation governing linear cosmological perturbations. As a result, enhancement effects of quan- 
tum corrections during long cosmic evolution times were suggested based on the observation that super-horizon curvature perturbations were not preserved, unlike classically, but had a growing mode. Other forms of perturbation equations were used, for instance, in [10, 11, 12, 13, 14]. Such effects may lead to observable imprints in the cosmic background radiation even though individual quantum-gravitational correction terms at sub-Planckian densities are small. The consideration, however, was restricted to the scalar mode in the longitudinal gauge, i.e. diagonal metric perturbations. While classically this procedure proves to be equivalent to a non-gauge fixed derivation, it leads to inconsistencies at the effective level in the presence of quantum corrections. Specifically, the effective equations resulting from the quantization of a gauge-fixed system in general are incompatible with each other, and gauge-fixing eliminates the freedom required to see systematically how the terms of consistent equations must be arranged. A non-gauge fixed treatment is thus necessary to evaluate all consistency conditions and to determine the gauge-invariant equation of motion for the curvature perturbation. (Similarly, if one uses only matter perturbations on a homogeneous gravitational background one is implicitly using a gauge-fixed treatment. This is in general inconsistent if no care is taken concerning the specific correction terms and the meaning of matter perturbations in relation to gauge-invariant quantities. Sometimes a "separate universe" picture [15, 16, 17] is used, arguing that at least for large scale modes quantum corrections to the homogeneous background equations should be sufficient to determine the evolution of inhomogeneities. But also here, as we will see in examples later, not all features will be visible based solely on homogeneous models without a full consistency analysis once quantum gravity corrections are included.)

A simple counting shows that the three independent functions describing scalar (gravity and matter) perturbations are subject to five equations of motion (see the Table 1). In general, such a system would be over-determined unless the equations are not independent. In gravity, they can be split into two types: i) evolution equations of second order in time derivatives and ii) constraint equations of lower order. Constraints restrict the initial data, and if they are preserved under evolution the system of equations is consistent. This is guaranteed automatically when equations of motion are obtained variationally from a covariant action, and is therefore satisfied for the classical equations. If quantum corrections are derived in a Hamiltonian approach, however, consistency is ensured only if the quantization is anomaly-free. The consistency of the resulting equations is thus tightly related to the closure of the constraint algebra. While the algebra of the constraints obtained from the classical Einstein-Hilbert action by a Legendre transformation is closed or, in Dirac's terms [18], the Hamiltonian and diffeomorphism constraints are of first class, this property may not sustain quantization. A consistent gauge invariant formulation of quantum-corrected equations of motion is possible only if the quantization is anomaly free, i.e. if the constraints remain first class.

As shown in [19, 20], standard loop quantization under very mild assumptions leads to a non-anomalous constraint algebra for vector and tensor modes. (At the linear level mode decomposition does not interfere with quantization, and quantum corrections to scalar, vector and tensor modes can be studied independently.) In [2] it was analyzed what types of (non-anomalous) quantum corrections are allowed for the scalar mode, obtaining the 
anomaly-freedom conditions ensuring a first class system. Once closure of the constraint algebra is provided, the formulation of gauge invariant equations of motion becomes possible, as developed in this paper.

We start with reviewing the correspondence between the canonical and covariant equations of motion, then derive the gauge invariant variables and finally obtain the gauge invariant quantum corrected linear Einstein equations. As we will see, consistency requires certain features of the corrected perturbation equations and of the gauge invariant variables, which could not be seen in gauge-fixed formulations. Several immediate consequences are discussed in the Sec. 5 and further in the conclusions, which also exhibit the final quantum corrected perturbation equations. Readers interested primarily in applications may turn directly to these sections.

\section{Basic variables and equations}

When a classical theory is quantized, the choice of basic variables often matters. While there are many equivalent formulations of classical physics, all related to each other by canonical transformations, such maps are rarely implementable as exact unitary transformations when quantized. This gives rise to different inequivalent quantizations of the same classical theory, and it can even prevent one from constructing a quantization in a particular classical formulation of the theory: there may be no Hilbert space representation where a certain choice of basic classical phase space variables will become well-defined operators.

In loop quantum gravity [3, 4, 5], the principle of background independence, which requires that well-defined operators do not refer to a metric other than the physical one to be turned into operators, distinguishes a special class of basic variables. In field theories such as general relativity, it is not field values at single points which can become well-defined operators, but only "smeared" versions obtained after integrating over spatial regions. Such integrations ensure that the operator-valued distributions, which field values would correspond to, become well-defined operators which can be multiplied to construct composite operators from them. Since the physical fields of canonical general relativity are spatial tensor fields, they cannot directly be integrated in a coordinate independent manner. Moreover, integration measures are not provided automatically because only the physical metric could be used on a curved manifold, but this metric itself is being turned into an operator. If one uses connection variables and densitized vector fields as canonical objects, however, their transformation properties ensure that they can be integrated over curves and surfaces, respectively, without requiring any additional integration measure. The resulting holonomies and fluxes then become well-defined operators in the quantum representation underlying loop quantum gravity [21].

This representation has characteristic properties which are implied by the choice of basic fields and their smearing. In particular, operators for spatial geometry such as the fluxes themselves or areas and volumes acquire discrete spectra [22, 23, 24]. This, in turn, determines how these basic operators can appear in composite ones such as Hamiltonians [25, 6, 7]. For instance, flux operators having discrete spectra containing the eigenvalue 
zero do not possess densely defined inverse operators. Since inverse triad components appear in Hamiltonians, the lack of a direct quantization entails quantum corrections in any effective Hamiltonian (see e.g. [26]), which will then also change the corresponding evolution as well as gauge properties. In this paper, we derive such equations precisely for corrections resulting from inverse triad components.

\subsection{Perturbed variables}

To do so, we perform the perturbation analysis of inhomogeneities in the basic variables underlying loop quantum gravity, such that we will be using primarily a densitized triad $E_{i}^{a}$ instead of the spatial metric $q_{a b}$ (satisfying $E_{i}^{a} E_{i}^{b}=q^{a b} \operatorname{det} q$ ). Moreover, in this canonical setting the remaining components $N$ and $N^{a}$ of the space-time metric

$$
\mathrm{d} s^{2}=-N^{2} \mathrm{~d} t^{2}+q_{a b}\left(\mathrm{~d} x^{a}+N^{a} \mathrm{~d} t\right)\left(\mathrm{d} x^{b}+N^{b} \mathrm{~d} t\right)
$$

will not be dynamical but play the role of Lagrange multipliers of constraints. In fact, their time derivatives do not appear in the Einstein-Hilbert action, which can be written in the canonical form

$$
S_{\mathrm{EH}}=\int \mathrm{d} t\left[\left(\frac{1}{8 \pi G} \int \mathrm{d}^{3} x \dot{K}_{a}^{i} E_{i}^{a}\right)-G_{\text {grav }}\left[\Lambda^{i}\right]-D_{\text {grav }}\left[N^{a}\right]-H_{\text {grav }}[N]\right]
$$

where $K_{a}^{i}$ is conjugate to $E_{i}^{a}$,

$$
\left\{K_{a}^{i}(x), E_{j}^{b}(y)\right\}=8 \pi G \delta_{a}^{b} \delta_{j}^{i} \delta^{3}(x, y),
$$

and related to extrinsic curvature $K_{a b}$ by $K_{a}^{i}=K_{a b} E_{i}^{b} / \sqrt{|\operatorname{det} E|}$. The remaining terms are the diffeomorphism constraint

$$
D_{\text {grav }}\left[N^{a}\right]=\frac{1}{8 \pi G} \int_{\Sigma} \mathrm{d}^{3} x N^{a}\left(\left(\partial_{a} K_{b}^{j}-\partial_{b} K_{a}^{j}\right) E_{j}^{b}-K_{a}^{j} \partial_{b} E_{j}^{b}\right)
$$

and the Hamiltonian constraint

$$
H_{\text {grav }}[N]=\frac{1}{16 \pi G} \int_{\Sigma} \mathrm{d}^{3} x N \frac{E_{i}^{a} E_{j}^{b}}{\sqrt{|\operatorname{det} E|}}\left(F_{a b}^{k} \epsilon_{k}^{i j}-2\left(1+\gamma^{2}\right) K_{a}^{[i} K_{b}^{j]}\right) .
$$

Here,

$$
F_{a b}^{k}=2 \partial_{[a}(\Gamma+\gamma K)_{b]}^{k}+\epsilon_{i j}^{k}(\Gamma+\gamma K)_{a}^{i}(\Gamma+\gamma K)_{b}^{j}
$$

is the curvature of the Ashtekar-Barbero connection [27, 28] $A_{a}^{i}=\Gamma_{a}^{i}+\gamma K_{a}^{i}$ defined in terms of the spin connection

$$
\Gamma_{a}^{i}=-\frac{1}{2} \epsilon^{i j k} E_{j}^{b}\left(\partial_{a} E_{b}^{k}-\partial_{b} E_{a}^{k}+E_{k}^{c} E_{a}^{l} \partial_{c} E_{b}^{l}-E_{a}^{k} \frac{\partial_{b}(\operatorname{det} E)}{\operatorname{det} E}\right)
$$


and $\gamma$ is the Barbero-Immirzi parameter [28, 29]. These variables also appear in the Gauss constraint

$$
G_{\text {grav }}\left[\Lambda^{i}\right]=\int \mathrm{d}^{3} x \Lambda^{i}\left(\partial_{a} E_{i}^{a}+\epsilon_{i j k} \Gamma_{a}^{j} E_{k}^{a}+\gamma \epsilon_{i j k} K_{a}^{j} E_{k}^{a}\right)
$$

in (2). This constraint will be solved explicitly by our parameterization of variables at the linear level, and its gauge will be fixed by a background triad. We can thus ignore it from now on.

For a perturbed metric of the form

$$
\mathrm{d} s^{2}=a^{2}(\eta)\left(-(1+2 \phi) \mathrm{d} \eta^{2}+2 \partial_{a} B \mathrm{~d} \eta \mathrm{d} x^{a}+\left((1-2 \psi) \delta_{a b}+2 \partial_{a} \partial_{b} E\right) \mathrm{d} x^{a} \mathrm{~d} x^{b}\right),
$$

as it describes general scalar perturbations $(\phi, \psi, E, B)$ around spatially flat FriedmannRobertson-Walker models in a general gauge, the background and perturbed triad in

$$
E_{i}^{a}=\bar{E}_{i}^{a}+\delta E_{i}^{a}
$$

are given by

$$
\bar{E}_{i}^{a}=\bar{p} \delta_{i}^{a} \equiv a^{2} \delta_{i}^{a} \quad, \quad \delta E_{i}^{a}=-2 \bar{p} \psi \delta_{i}^{a}+\bar{p}\left(\delta_{i}^{a} \Delta-\partial^{a} \partial_{i}\right) E,
$$

where $\Delta$ is the Laplace operator on flat space, indices run from 1 to $3, a$ is the (background) scale factor and $\psi$ and $E$ describe the spatial part of the perturbed metric. (We use standard notations where $E$ is one of the scalar modes distinct from the full densitized triad $E_{i}^{a}$. The latter will always be written with indices such that no confusion should arise.) The other two scalar metric perturbations $\phi$ and $B$ are related to the perturbed lapse function and shift vector respectively

$$
\delta N=\bar{N} \phi \quad, \quad N^{a}=\partial^{a} B
$$

and will enter the extrinsic curvature components, also perturbed as

$$
K_{a}^{i}=\bar{K}_{a}^{i}+\delta K_{a}^{i}=\bar{k} \delta_{a}^{i}+\delta K_{a}^{i}
$$

This splitting, with the condition that $\delta E_{i}^{a}$ and $\delta K_{a}^{i}$ do not have homogeneous modes in order to avoid double counting, results in Poisson brackets

$$
\{\bar{k}, \bar{p}\}=\frac{8 \pi G}{3 V_{0}} \quad, \quad\left\{\delta K_{a}^{i}(x), \delta E_{j}^{b}(y)\right\}=8 \pi G \delta_{j}^{i} \delta_{a}^{b} \delta^{3}(x-y) .
$$

The homogeneous mode is defined by

$$
\bar{p}=\frac{1}{3 V_{0}} \int E_{i}^{a} \delta_{a}^{i} \mathrm{~d}^{3} x \quad, \quad \bar{k}=\frac{1}{3 V_{0}} \int K_{a}^{i} \delta_{i}^{a} \mathrm{~d}^{3} x
$$

where we integrate over a bounded region of coordinate size $V_{0}=\int \mathrm{d}^{3} x$ which could be over the whole space if it is compact, or a sufficiently large region encompassing all the scales of perturbations of interest. Although $V_{0}$, which depends on coordinates as well as 
the choice we make for the integration region, enters the definition of variables and their Poisson structure, particular correction terms for observables will not depend on its value. Using the homogeneous modes, perturbations are defined by (8) and (11).

The specific form of $\bar{K}_{a}^{i}$ and $\delta K_{a}^{i}$ in relation to time derivatives of $\bar{p}$ and $\delta E_{i}^{a}$, analogously to the triad fields in (9), follows from the equations of motion. Before deriving these relations we thus introduce the quantum corrections we consider because they have a bearing on the form of components of $K_{a}^{i}$.

\subsection{Quantum corrections}

The Hamiltonian constraint (5) contains a factor of an inverse determinant of the densitized triad. This inverse cannot be quantized directly because the integrated determinant itself is quantized to an operator with zero in the discrete spectrum, precluding the existence of an inverse operator. Nevertheless, well-defined operators quantizing (5), including the inverse triad, exist [6, 30]. However, the behavior of expectation values of the operators differs on small length scales from the classical behavior even in semiclassical states, which implies the presence of a correction function $\alpha$ multiplying the Hamiltonian density. This function must be scalar (of density weight zero) to ensure the proper behavior of the integral, and it can depend functionally on all the phase space variables in possibly non-local ways. Such a general dependence would make an analysis of the constraint algebra and of equations of motion intractable, and so we have organized the calculations in [2] by first assuming a primary correction function $\alpha\left(E_{i}^{a}\right)$ which depends only on the triad and does so only in algebraic form. By itself, this does not produce anomaly-free quantizations, which however do exist if additional counter-terms are added containing new correction functions whose relation to the primary correction is fixed by anomaly-cancellation. These extra terms can be interpreted as arising from a more complicated dependence of $\alpha$ on all the phase space variables, which is derived systematically by this process.

Specifically, the quantum corrected Hamiltonian constraint derived in [2] can conveniently be written as

$$
H^{Q}=H_{\text {grav }}^{Q}[\bar{N}]+H_{\text {grav }}^{Q}[\delta N]+H_{\text {matt }}^{Q}[\bar{N}]+H_{\text {matt }}^{Q}[\delta N]
$$

where the gravitational part is expanded by powers of inhomogeneities $\delta E_{i}^{a}, \delta K_{a}^{i}$ and $\delta N$ as

$$
\begin{aligned}
H_{\text {grav }}^{Q}[\bar{N}] & :=\frac{1}{16 \pi G} \int \mathrm{d}^{3} x \bar{N}\left[\bar{\alpha} \mathcal{H}^{Q(0)}+\alpha^{(2)} \mathcal{H}^{Q(0)}+\bar{\alpha} \mathcal{H}^{Q(2)}\right], \\
H_{\text {grav }}^{Q}[\delta N] & :=\frac{1}{16 \pi G} \int \mathrm{d}^{3} x \delta N\left[\bar{\alpha} \mathcal{H}^{Q(1)}\right]
\end{aligned}
$$

and the matter Hamiltonian reads

$$
\begin{aligned}
H_{\text {matter }}^{Q}[\bar{N}] & =\int_{\Sigma} \mathrm{d}^{3} x \bar{N}\left[\left(\bar{\nu} \mathcal{H}_{\pi}^{Q(0)}+\mathcal{H}_{\varphi}^{Q(0)}\right)+\left(\nu^{(2)} \mathcal{H}_{\pi}^{Q(0)}+\bar{\nu} \mathcal{H}_{\pi}^{Q(2)}+\bar{\sigma} \mathcal{H}_{\nabla}^{Q(2)}+\mathcal{H}_{\varphi}^{Q(2)}\right)\right] \\
H_{\text {matter }}^{Q}[\delta N] & =\int \mathrm{d}^{3} x \delta N\left[\bar{\nu} \mathcal{H}_{\pi}^{Q(1)}+\mathcal{H}_{\varphi}^{Q(1)}\right] .
\end{aligned}
$$


In all expansions, a bar is used to denote background quantities while superscripts indicate the inhomogeneous order. The Hamiltonian densities in the expansions are given by [2]

$$
\begin{aligned}
\mathcal{H}^{Q(0)}= & -6 \bar{k}^{2} \sqrt{\bar{p}}, \\
\mathcal{H}^{Q(1)}= & -4(1+f) \bar{k} \sqrt{\bar{p}} \delta_{j}^{c} \delta K_{c}^{j}-(1+g) \frac{\bar{k}^{2}}{\sqrt{\bar{p}}} \delta_{c}^{j} \delta E_{j}^{c}+\frac{2}{\sqrt{\bar{p}}} \partial_{c} \partial^{j} \delta E_{j}^{c}, \\
\mathcal{H}^{Q(2)}= & \sqrt{\bar{p}} \delta K_{c}^{j} \delta K_{d}^{k} \delta_{k}^{c} \delta_{j}^{d}-\sqrt{\bar{p}}\left(\delta K_{c}^{j} \delta_{j}^{c}\right)^{2}-\frac{2 \bar{k}}{\sqrt{\bar{p}}} \delta E_{j}^{c} \delta K_{c}^{j} \\
& \quad-\frac{\bar{k}^{2}}{2 \bar{p}^{3 / 2}} \delta E_{j}^{c} \delta E_{k}^{d} \delta_{c}^{k} \delta_{d}^{j}+\frac{\bar{k}^{2}}{4 \bar{p}^{3 / 2}}\left(\delta E_{j}^{c} \delta_{c}^{j}\right)^{2}-(1+h) \frac{\delta^{j k}}{2 \bar{p}^{3 / 2}}\left(\partial_{c} \delta E_{j}^{c}\right)\left(\partial_{d} \delta E_{k}^{d}\right) .
\end{aligned}
$$

for gravity and by

$$
\begin{aligned}
\mathcal{H}_{\pi}^{Q(0)} & =\frac{\bar{\pi}_{\bar{\varphi}}^{2}}{2 \bar{p}^{3 / 2}}, \quad \mathcal{H}_{\nabla}^{Q(0)}=0 \quad, \quad \mathcal{H}_{\varphi}^{Q(0)}=\bar{p}^{3 / 2} V(\bar{\varphi}) \\
\mathcal{H}_{\pi}^{Q(1)} & =\left(1+f_{1}\right) \frac{\bar{\pi} \delta \pi}{\bar{p}^{3 / 2}}-\left(1+f_{2}\right) \frac{\bar{\pi}^{2}}{2 \bar{p}^{3 / 2}} \frac{\delta_{c}^{j} \delta E_{j}^{c}}{2 \bar{p}} \\
\mathcal{H}_{\nabla}^{Q(1)} & =0 \\
\mathcal{H}_{\varphi}^{Q(1)} & =\bar{p}^{3 / 2}\left(\left(1+f_{3}\right) V_{, \varphi}(\bar{\varphi}) \delta \varphi+V(\bar{\varphi}) \frac{\delta_{c}^{j} \delta E_{j}^{c}}{2 \bar{p}}\right) \\
\mathcal{H}_{\pi}^{Q(2)} & =\left(1+g_{1}\right) \frac{\delta \pi^{2}}{2 \bar{p}^{3 / 2}}-\left(1+g_{2}\right) \frac{\bar{\pi} \delta \pi}{\bar{p}^{3 / 2}} \frac{\delta_{c}^{j} \delta E_{j}^{c}}{2 \bar{p}}+\frac{1}{2} \frac{\bar{\pi}^{2}}{\bar{p}^{3 / 2}}\left(\left(1+g_{3}\right) \frac{\left(\delta_{c}^{j} \delta E_{j}^{c}\right)^{2}}{8 \bar{p}^{2}}+\frac{\delta_{c}^{k} \delta_{d}^{j} \delta E_{j}^{c} \delta E_{k}^{d}}{4 \bar{p}^{2}}\right) \\
\mathcal{H}_{\nabla}^{Q(2)} & =\frac{1}{2}\left(1+g_{5}\right) \sqrt{\bar{p}} \delta^{a b} \partial_{a} \delta \varphi \partial_{b} \delta \varphi \\
\mathcal{H}_{\varphi}^{Q(2)} & =\bar{p}^{3 / 2}\left[\left(1+g_{6}\right) \frac{1}{2} V_{, \varphi \varphi}(\bar{\varphi}) \delta \varphi^{2}+V_{, \varphi}(\bar{\varphi}) \delta \varphi \frac{\delta_{c}^{j} \delta E_{j}^{c}}{2 \bar{p}}+V(\bar{\varphi})\left(\frac{\left(\delta_{c}^{j} \delta E_{j}^{c}\right)^{2}}{8 \bar{p}^{2}}-\frac{\delta_{c}^{k} \delta_{d}^{j} \delta E_{j}^{c} \delta E_{k}^{d}}{4 \bar{p}^{2}}\right)\right] .
\end{aligned}
$$

for matter.

The $\bar{p}$-dependent functions $\bar{\alpha}, \bar{\nu}$ and $\bar{\sigma}$ are primary correction functions whose origin is the presence of inverse triad operators in a constraint operator. Their form can be computed in isotropic models [30, 31, 32] or with certain gauge assumptions for inhomogeneous states [33, 26]. Classically, we have $\bar{\alpha}=\bar{\nu}=\bar{\sigma}=1$, while there can be strong deviations from this value for small values of elementary flux variables which quantize the densitized triad. This deep quantum regime is difficult to control, however, and the derivations in [2] of an anomaly-free constraint algebra are valid for primary correction functions of the form

$$
\bar{\alpha}(a)=1+c_{\alpha}\left(\frac{\ell_{\mathrm{P}}^{2}}{a^{2}}\right)^{n_{\alpha}}+\cdots
$$

which are perturbative in the Planck length $\ell_{\mathrm{P}}=\sqrt{G \hbar}$, i.e. $n_{\alpha}>0$. Explicit values for coefficients $c_{\alpha}, c_{\nu}$ and $c_{\sigma}$, which are generically positive such that $\bar{\alpha}(a)>1$ in perturbative 
regimes, as well as the exponents $n_{\alpha}, n_{\nu}$ and $n_{\sigma}$ can be derived from specific quantizations, but they are subject to quantization ambiguities 1 One purpose of deriving anomaly-free versions of the constraints is to provide consistency conditions among some of these values, fixing some quantization ambiguities.

For an anomaly-free quantization in a gauge-independent manner, the presence of these primary correction functions requires counter-terms with coefficients $f, g$ and $h$ as well as $f_{i}$ and $g_{i}$ which also depend on $\bar{p}$ in a way fixed by anomaly cancellation conditions. For the situation under consideration where the matter sector consists of a scalar field with a non-trivial potential, we have

$$
\begin{aligned}
2 f^{\prime} \bar{p} & =-\frac{\bar{\alpha}^{\prime} \bar{p}}{\bar{\alpha}} \\
g & =-2 f \\
f_{1} & =f-\frac{\bar{\nu}^{\prime} \bar{p}}{3 \bar{\nu}}
\end{aligned}
$$

and

$$
\frac{\partial \alpha^{(2)}}{\partial\left(\delta E_{i}^{a}\right)}\left(\delta_{j}^{c} \delta_{i}^{a}-\delta_{j}^{a} \delta_{i}^{c}\right)=\frac{\alpha^{\prime}}{3 p} \delta E_{j}^{c}, \quad \frac{\partial \nu^{(2)}}{\partial\left(\delta E_{i}^{a}\right)}\left(\delta_{j}^{c} \delta_{i}^{a}-\delta_{j}^{a} \delta_{i}^{c}\right)=\frac{\nu^{\prime}}{3 p} \delta E_{j}^{c} .
$$

Here and in what follows, primes denote derivatives by $\bar{p}$. Moreover, we have

$$
\bar{\alpha}^{2}=\bar{\nu} \bar{\sigma}
$$

Other consistency conditions will be recalled later from [2] (also discussed in Appendix B) whenever they are being used. Classically, all counter-terms vanish, e.g. $f=f_{1}=g=0$. With the consistency conditions the system of corrected constraints is anomaly-free to the perturbative orders considered, which is linear in inhomogeneities (requiring second order expansions of the constraints which generate linear equations of motion) as well as leading order in the corrections of (19) . The latter assumption of perturbativity implies that we ignore terms such as $(\bar{\alpha}-1)^{2},(p \mathrm{~d} \bar{\alpha} / \mathrm{d} p)^{2}$ or $f^{2}$ compared to $\bar{\alpha}-1$.

With the corrected Hamiltonian, we can derive the equations of motion it generates. From $\dot{\bar{p}}=\left\{\bar{p}, H_{\mathrm{grav}}^{Q}[N]\right\}$, for instance, we obtain the background part $\bar{K}_{a}^{i}=\bar{k} \delta_{a}^{i}$ of extrinsic curvature where $\bar{k}$ is related to the conformal Hubble parameter by

$$
\bar{\alpha} \bar{k}=\mathscr{H} \equiv \frac{\dot{\bar{p}}}{2 \bar{p}}
$$

\footnotetext{
${ }^{1}$ Even the isotropic quantization used for the background evolution is subject to quantization ambiguities. Uniqueness results of the quantum dynamics can be obtained only based on ad-hoc assumptions, and they sometimes occur as a result of incorrect implementations of quantization schemes. The source of ambiguities is the representation of operators, such as inverse triad operators used here, but also the underlying refinement behavior of a discrete state underlying the quantum evolution [34, 35]; see also [36] and the appendix of [2]. Both ingredients combine to determine the values of $c_{\alpha}$ and $n_{\alpha}$. Note also that $c_{\alpha}$, when correctly derived using lattice refinement, is coordinate dependent in such a way that the combination with the scale factor in (19) is scaling independent.
} 
The choice of the background lapse function $\bar{N}=a$, used to derive (25), corresponds to the conformal time $\eta$ whose derivative we denote by a dot. In general, the total time derivative of an arbitrary phase space function is given by its Poisson bracket with $H^{Q}[N]+$ $D\left[N^{a}\right]$ parameterized by the total lapse $N \equiv \bar{N}+\delta N$ and shift $N^{a} \equiv \bar{N}^{a}+\delta N^{a}$. Nonetheless, for a background quantity the Poisson bracket above, using only $H^{Q}[\bar{N}]$, coincides with the conformal (background) time derivative up to the second perturbative order.

Similarly, the form of the perturbation $\delta K_{a}^{i}$ can be deduced from Hamilton's equation for $\delta \dot{E}_{i}^{a}$. Namely, using

$$
\delta \dot{E}_{i}^{a} \equiv\left\{\delta E_{i}^{a}, H^{Q}[N]+D\left[N^{a}\right]\right\}
$$

along with (9) and (25), we obtain

$$
\bar{\alpha} \delta K_{a}^{i}=-\delta_{a}^{i}[\dot{\psi}+\mathscr{H}(\psi+\phi(1+f))]+\partial_{a} \partial^{i}[\mathscr{H} E-(B-\dot{E})],
$$

where the counter-term $f(\bar{p})$ appears.

Matter is represented by a scalar field $\varphi=\bar{\varphi}+\delta \varphi$ with potential $V(\varphi)$ and its conjugate momentum $\pi=\bar{\pi}+\delta \pi$. As before, we use the equations of motion

$$
\dot{\bar{\varphi}} \equiv\left\{\bar{\varphi}, H^{Q}[N]+D\left[N^{a}\right]\right\}, \quad \delta \dot{\varphi} \equiv\left\{\delta \varphi, H^{Q}[N]+D\left[N^{a}\right]\right\}
$$

to express the field momentum as

$$
\bar{\pi}=\dot{\bar{\varphi}} \frac{\bar{p}}{\bar{\nu}}, \quad \delta \pi=\frac{\bar{p}}{\bar{\nu}}\left(\left(\delta \dot{\varphi}-\dot{\bar{\varphi}}\left(1+f_{1}\right) \phi\right)\left(1-g_{1}\right)+\dot{\bar{\varphi}} \frac{\delta E_{i}^{a} \delta_{a}^{i}}{2 \bar{p}}\right) .
$$

Before proceeding to gauge transformations in the next section we note the relation between the canonical and covariant equations of motion, summarized in Table 11. There are three background equations, only two of which are independent, for the two unknown functions: scale factor $a(\eta)$ and matter scalar field $\bar{\varphi}(\eta)$ depending on the conformal time $\eta$. Those are the Friedmann, Raychaudhuri and Klein-Gordon equations [26]

$$
\begin{aligned}
\mathscr{H}^{2} & =\frac{8 \pi G}{3} \bar{\alpha}\left(\frac{\dot{\bar{\varphi}}^{2}}{2 \bar{\nu}}+\bar{p} V(\bar{\varphi})\right), \\
\ddot{\mathscr{C}} & =\mathscr{H}^{2}\left(1+\frac{\bar{\alpha}^{\prime} \bar{p}}{\bar{\alpha}}\right)-4 \pi G \frac{\bar{\alpha}}{\bar{\nu}} \dot{\bar{\varphi}}^{2}\left(1-\frac{\bar{\nu}^{\prime} \bar{p}}{3 \bar{\nu}}\right), \\
\ddot{\bar{\varphi}} & +2 \mathscr{H} \dot{\bar{\varphi}}\left(1-\frac{\bar{\nu}^{\prime} \bar{p}}{\bar{\nu}}\right)+\bar{\nu} \bar{p} V_{, \varphi}(\bar{\varphi})=0,
\end{aligned}
$$

where the prime indicates a derivative with respect to $\bar{p}$. These equations are listed in the left column and correspond to the background Hamiltonian constraint and two pairs of dynamical (Hamilton's) equations. Each pair of the first order equations is to be combined into a single second order equation.

More generally, covariant equations which are less than second order (with respect to the conformal time derivative) correspond to constraint equations in the canonical formalism. 


\begin{tabular}{|c|c|}
\hline Covariant Equations & Canonical Equations \\
\hline Background Friedmann & Background Hamiltonian Constraint \\
\hline Background Raychaudhuri & $\overline{\bar{k}} \& \dot{\bar{p}}$ \\
\hline Background Klein-Gordon & $\dot{\bar{\varphi}} \& \dot{\bar{\pi}}$ \\
\hline Perturbed Einstein ${ }_{T}^{T}$ & Perturbed Hamiltonian Constraint \\
\hline Perturbed Einstein ${ }_{T}^{S}$ & Perturbed Diffeomorphism Constraint \\
\hline Perturbed Einstein ${ }_{S}^{S}$ & $\delta \dot{K} \& \delta \dot{E}$ \\
\hline Perturbed Klein-Gordon & $\delta \dot{\varphi} \& \delta \dot{\pi}$ \\
\hline
\end{tabular}

Table 1: Table of correspondence between the background and perturbed canonical equations and the covariant equations of general relativity. In the covariant column, the subscripts ' $\mathrm{S}$ ' and ' $\mathrm{T}$ ' stand for spatial and temporal components respectively. In the canonical framework, equations are of two types: constraint equations and dynamical (Hamilton's) equations for the time derivatives of canonical pairs. Note that both 'SS'- and ' $\delta \dot{K} \&$ $\delta \dot{E}^{\prime}$-equations are tensorial. Indices of $\delta K_{a}^{i}$ and $\delta E_{i}^{a}$ have been suppressed for simplicity.

They should be viewed as restrictions on the initial conditions. As mentioned earlier, for consistent and unambiguous (gauge-invariant) evolution such constraints must be preserved by the dynamical equations. In the canonical language, this property of constraints is translated into the requirement of closure of the constraint algebra, as it is analyzed for the corrected constraints in [2]. As a result, relations such as (201), (22), (21) and (23) between the correction functions have to be satisfied as conditions for higher order terms of primary correction functions.

\section{Gauge transformations}

In classical relativity, it is the Lie derivative which provides the form of gauge transformations of the fields such as metric components, corresponding to changes of coordinates. After quantization it is no longer clear what the analog of these gauge transformations should be, given that the underlying space-time notion would have to be determined from the quantum theory itself. In most approaches to quantum gravity, one does not expect the fundamental space-time picture to be described by a smooth manifold. Here, an advantage of the canonical formulation is that gauge transformations are directly generated as Poisson brackets of the fields with the constraints. Classically, this reproduces the formulas obtained by Lie derivatives, and it can directly be extended to canonical quantum gravity capturing changes to the quantum space-time structure. With corrections (14) to the classical constraints (5), it is not only equations of motion but also the form of gauge transformations which changes. Thus, gauge invariant combinations of the perturbations take different forms than they do classically.

However, as we saw not all the space-time metric components are dynamical phase space variables, and only the gauge transformations for the spatial metric, or $E_{i}^{a}$ and $K_{a}^{i}$, 
will be determined straightforwardly. In this section we derive these transformations and show how also the transformations of the remaining components $N$ and $N^{a}$, or $\phi$ and $B$ in the scalar perturbations related to the Lagrange multipliers, can be obtained.

\subsection{Classical gauge-invariant variables}

It is instructive to introduce the canonical derivations and required notions in the classical case first, after which we will directly extend the expressions to those including quantum corrections. (Canonical treatments of classical perturbations have also been discussed in [37, 38, 39].)

In the covariant formulation, gauge transformations constitute local infinitesimal coordinate transformations

$$
x^{\mu} \rightarrow \tilde{x}^{\mu}=x^{\mu}+\xi^{\mu}(x),
$$

generated by vector fields $\xi^{\mu}$. In a perturbative setting, the infinitesimal field $\xi^{\mu}$ will be treated as a first order perturbation. Under this coordinate transformation any tensor field receives a correction equal to its Lie derivative along $\xi^{\mu}$. The part of the transformation relevant for the scalar mode can be parameterized by two scalar functions $\xi^{0}$ and $\xi$ such that

$$
\xi^{\mu}=\left(\xi^{0}, \partial^{a} \xi\right)
$$

where $a$ indicates a spatial direction 1,2 or 3 .

There are four (spatial) scalar perturbations in a space-time metric, $\phi, \psi, B$ and $E$ as they appear in the line element (7) in conformal time $\eta$. These perturbations are subject to two independent gauge transformations by $\xi^{0}$ and $\xi$. We now briefly recall how these transformations follow from changes of coordinates to verify later that the classical canonical transformations produce the correct form.

If only $\xi^{0}$ is non-zero, the coordinate transformation changes $\eta$ to $\eta+\xi^{0}$, which for $\mathrm{d} \eta^{2}$ implies, to first order in $\xi^{0}$,

$$
\mathrm{d}\left(\eta+\xi^{0}\right)^{2}=\mathrm{d} \eta^{2}+2 \dot{\xi}^{0} \mathrm{~d} \eta^{2}+2 \xi^{0},{ }_{a} \mathrm{~d} \eta \mathrm{d} x^{a}
$$

and $a(\eta)^{2}$ changes to $a(\eta)^{2}\left(1+2 \dot{a} \xi^{0} / a\right)$. Inserting this in (7), we read off the transformation formulas

$$
\phi \mapsto \phi+\dot{\xi}^{0}+\mathscr{H} \xi^{0} \quad, \quad \psi \mapsto \psi-\mathscr{H} \xi^{0} \quad, \quad B \mapsto B-\xi^{0} \quad, \quad E \mapsto E
$$

where $\mathscr{H}=\dot{a} / a$ as in (25).

If only $\xi$ is non-zero, $\mathrm{d} x_{a} \mathrm{~d} x^{a}$ changes to

$$
\mathrm{d}\left(x_{a}+\xi_{, a}\right) \mathrm{d}\left(x^{a}+\xi,^{a}\right)=\mathrm{d} x_{a} \mathrm{~d} x^{a}+2 \dot{\xi}_{, a} \mathrm{~d} \eta \mathrm{d} x^{a}+2 \xi,_{a b} \mathrm{~d} x^{a} \mathrm{~d} x^{b}
$$

which yields

$$
\phi \mapsto \phi \quad, \quad \psi \mapsto \psi \quad, \quad B \mapsto B+\dot{\xi} \quad, \quad E \mapsto E+\xi
$$


We thus see that $B-\dot{E}$ is invariant under $\xi$-transformations (or spatial diffeomorphisms) and changes to $B-\dot{E}-\xi^{0}$ under $\xi^{0}$-transformations. Thus, the Bardeen variables [40]

$$
\Phi=\phi+\mathscr{H}(B-\dot{E})+(B-\dot{E}) \quad \text { and } \quad \Psi=\psi-\mathscr{H}(B-\dot{E})
$$

are gauge invariant. For a scalar field $\varphi$, the only change is under $\xi^{0}$ and given by $\delta \varphi+\dot{\bar{\varphi}} \xi^{0}$. Here,

$$
\delta \varphi^{\mathrm{GI}}=\delta \varphi+\dot{\bar{\varphi}}(B-\dot{E})
$$

is gauge invariant.

In the canonical formulation, gauge transformations are generated by the Hamiltonian and diffeomorphism constraints. The corresponding lapse function and shift vector to be inserted are also first order perturbations related to the infinitesimal vector field $\xi^{\mu}$ via

$$
\delta N=\bar{N} \xi_{0}, \quad \delta N^{a}=\partial^{a} \xi
$$

which follow from the metric decomposition (11) in terms of the spatial metric $q_{a b}$, lapse function $N$ and shift vector $N^{a}$. With first order smearing functions, the gauge-generating constraints are at least of second perturbative order. From now on we will denote the gauge transformations of a phase space quantity $X$ as

$$
\delta_{\left[\xi_{0}, \xi\right]} X \equiv\left\{X, H^{(2)}\left[\bar{N} \xi_{0}\right]\right\}+\left\{X, D^{(2)}\left[\partial^{a} \xi\right]\right\}
$$

where

$$
H^{(2)}[\delta N]=\frac{1}{16 \pi G} \int \mathrm{d}^{3} x \delta N\left[\mathcal{H}^{(1)}+16 \pi G\left(\mathcal{H}_{\pi}^{(1)}+\mathcal{H}_{\varphi}^{(1)}\right)\right]
$$

with the Hamiltonian densities given by

$$
\mathcal{H}^{(1)}=-4 \bar{k} \sqrt{\bar{p}} \delta_{j}^{c} \delta K_{c}^{j}-\frac{\bar{k}^{2}}{\sqrt{\bar{p}}} \delta_{c}^{j} \delta E_{j}^{c}+\frac{2}{\sqrt{\bar{p}}} \partial_{c} \partial^{j} \delta E_{j}^{c}
$$

and

$$
\mathcal{H}_{\pi}^{(1)}=\frac{\bar{\pi} \delta \pi}{\bar{p}^{3 / 2}}-\frac{\bar{\pi}^{2}}{2 \bar{p}^{3 / 2}} \frac{\delta_{c}^{j} \delta E_{j}^{c}}{2 \bar{p}}, \quad \mathcal{H}_{\nabla}^{(1)}=0, \quad \mathcal{H}_{\varphi}^{(1)}=\bar{p}^{3 / 2}\left(V_{, \varphi}(\bar{\varphi}) \delta \varphi+V(\bar{\varphi}) \frac{\delta_{c}^{j} \delta E_{j}^{c}}{2 \bar{p}}\right) .
$$

For the diffeomorphism constraint (44), we have the second order term

$$
D^{(2)}\left[\delta N^{a}\right]=\frac{1}{8 \pi G} \int_{\Sigma} \mathrm{d}^{3} x \delta N^{a}\left[\bar{p} \partial_{a}\left(\delta_{k}^{d} \delta K_{d}^{k}\right)-\bar{p}\left(\partial_{k} \delta K_{a}^{k}\right)-\bar{k} \delta_{a}^{k}\left(\partial_{d} \delta E_{k}^{d}\right)+8 \pi G\left(\bar{\pi} \partial_{a} \delta \varphi\right)\right]
$$

In what follows, we perform canonical gauge transformations on the basic phase variables and demonstrate that this, too, results in the gauge invariant combinations (35). 


\subsubsection{Gauge transformations of basic variables}

We start by computing gauge transformations of the basic phase space variables: $K_{a}^{i}, E_{i}^{a}$, $\varphi$ and $\pi$. Using (38) with the constraints (39) and (42) we obtain

$$
\begin{aligned}
\delta_{\left[\xi_{0}, \xi\right]} \delta K_{a}^{i} & =\partial^{i} \partial_{a}\left(\xi_{0}+\bar{k} \xi\right)-\frac{\bar{k}^{2}}{2} \xi_{0} \delta_{a}^{i}+4 \pi G\left[\bar{p} V(\bar{\varphi})-\frac{\dot{\varphi}^{2}}{2}\right] \xi_{0} \delta_{a}^{i} \\
\delta_{\left[\xi_{0}, \xi\right]} \delta E_{i}^{a} & =2 \bar{k} \bar{p} \xi_{0} \delta_{i}^{a}+\bar{p}\left(\delta_{i}^{a} \Delta \xi-\partial^{a} \partial_{i} \xi\right) \\
\delta_{\left[\xi_{0}, \xi\right]} \delta \varphi & =\overline{\bar{p}} \xi_{0} \\
\delta_{\left[\xi_{0}, \xi\right]} \delta \pi & =\bar{\pi} \Delta \xi-\bar{p}^{2} V^{\prime}(\bar{\varphi}) \xi_{0}
\end{aligned}
$$

for the basic gravity and matter perturbations. Note that the background lapse function has again been set to $\bar{N}=\sqrt{\bar{p}}$ for gauge transformations of a metric in conformal time. It is also easy to see that when acting upon the background quantities $\bar{k}, \bar{p}, \bar{\varphi}$, and $\bar{\pi}$ these gauge transformations will generate only second order contributions. Hence in the equations of motion the background phase space variables can be treated as gauge-invariant up to the desired order.

The perturbative treatment introduces a subtlety in the interpretation of transformations: In the unperturbed case, the gauge transformation of a phase space function $X(K, E, \varphi, \pi)$ generated by the diffeomorphism constraint acts as a Lie derivative

$$
\left\{X, D\left[\xi^{a}\right]\right\}=\mathcal{L}_{\vec{\xi}} X
$$

along the vector field $\xi^{a}$. At the same time, the diffeomorphism transformation of the perturbations is given by the $\xi$-part of (43), i.e. $\left\{\delta X, D\left[\xi^{a}\right]\right\}=\delta_{[0, \xi]} \delta X$, whereas the barred quantities remain intact at the linear level $\left\{\bar{X}, D\left[\xi^{a}\right]\right\}=O(2)$. For a scalar field, these Poisson brackets can directly be identified as Lie derivatives of background quantities and perturbations:

$$
\mathcal{L}_{\vec{\xi}} \bar{\varphi}=\xi^{a} \partial_{a} \bar{\varphi}=0 \quad, \quad \mathcal{L}_{\vec{\xi}} \delta \varphi=\xi^{a} \partial_{a} \delta \varphi=O(2) .
$$

However, if one computes the Lie derivatives of perturbative terms of a tensorial object, or even of a scalar of non-zero density weight, one can notice that they do not coincide with the $\xi$-part of (43). For instance,

$$
\mathcal{L}_{\bar{\xi}} \bar{\pi}=\xi^{a} \partial_{a} \bar{\pi}+\bar{\pi} \partial_{a} \xi^{a}=\bar{\pi} \partial_{a} \xi^{a} \quad, \quad \mathcal{L}_{\bar{\xi}} \delta \pi=\xi^{a} \partial_{a} \delta \pi+\delta \pi \partial_{a} \xi^{a}=O(2)
$$

while

$$
\delta_{[0, \xi]} \bar{\pi}=0 \quad, \quad \delta_{[0, \xi]} \delta \pi=\bar{\pi} \Delta \xi=\bar{\pi} \partial_{a} \xi^{a}
$$

for $\xi^{a}=\partial^{a} \xi$. Similar discrepancies occur for the triad and extrinsic curvature.

Nevertheless, gauge transformations are related to the Lie derivative. As shown in App. A of [2], up to a higher perturbative order we have

$$
\left\{\bar{X}, D\left[\xi^{a}\right]\right\}=\left[\mathcal{L}_{\vec{\xi}} X\right]^{(0)}, \quad\left\{\delta X, D^{(2)}\left[\xi^{a}\right]\right\}=\left[\mathcal{L}_{\vec{\xi}} X\right]^{(1)}
$$


for the background and perturbed parts of $X$ respectively. (As explained in more detail in [2], the second Poisson bracket takes into account the fact that perturbations $\delta X$ do not contain zero modes.) These two equations above are consistent with the Lie derivative of the full variable $X$

$$
\left\{\bar{X}+\delta X, D\left[\xi^{a}\right]\right\}=\mathcal{L}_{\vec{\xi}}(\bar{X}+\delta X) .
$$

Individual terms in this expansion, when computed either via (44) or via (38), do not agree in general because $\left(\mathcal{L}_{\vec{\xi}} X\right)^{(0)}$ may not equal $\mathcal{L}_{\vec{\xi}}\left(X^{(0)}\right)=\mathcal{L}_{\vec{\xi}} \bar{X}$ : While the $\xi$-gauge transformation of an unperturbed variable is equivalent to taking a Lie derivative, the perturbation procedure breaks this equivalence. For instance, the Lie derivative of a background quantity (being linear) contributes to the gauge-transformation of its perturbation, not of the background quantity itself. At the same time, the Lie derivative of a linear perturbation (being at least a quadratic quantity and hence neglected here) contributes to the back-reaction on the background. Their zero- and first-order parts of the Lie derivative do contribute to the diffeomorphism transformation, with combined contribution equal to the diffeomorphism transformation of the full variable, although in a rather mixed way. When combined to $\bar{X}+\delta X$, conventional transformations are obtained.

\subsubsection{Transformation of the lapse function and shift vector}

In the covariant formulation, the lapse function and shift vector are merely components of the space-time metric, and hence subject to coordinate (gauge) transformations in the same way as any other metric component. In the Hamiltonian framework, on the other hand, lapse and shift act as Lagrange multipliers and are not phase space variables. Therefore, unlike e.g. triad components, their gauge transformations cannot be directly obtained as Poisson brackets (38) (which would always give zero) with the gauge-generating constraints. Nevertheless, there exists an indirect procedure 2

A coordinate change causes a change in the space-time foliation by spatial slices, and thus the induced $E_{i}^{a}$ and $K_{a}^{i}$ change according to their gauge transformations. Since the slicing is determined by lapse and shift they, too, must change. Lapse and shift not only determine the slicing but also, as in (26) and (28), equations of motion which triad and extrinsic curvature have to satisfy as one moves from one slice to the next. Consistency of equations of motion for the gauge-transformed canonical variables thus requires certain transformations of the lapse and shift: They have to change such that they generate the correct equations of motion for the transformed $E_{i}^{a}$ and $K_{a}^{i}$, on which a canonical gauge transformation has been applied. In this way, gauge transformations for $N$ and $N^{a}$ result unambiguously, even though these are not phase space variables.

Hamilton's equations such as (26) have a time derivative of a phase space variable on their left hand side, while the right hand side depends on phase space variables and the Lagrange multipliers. Thus performing a gauge transformation on both sides of the equations one can obtain the transformations of $\delta N$ and $\delta N^{a}$. The non-trivial part of this

\footnotetext{
${ }^{2}$ Alternatively, Poisson brackets can be defined on an extended phase space which also includes the Lagrange multipliers [41].
} 
recipe is the gauge transformation of the left hand side, as a time derivative of a phase space variable is not itself a phase space variable, and hence its Poisson bracket with constraints is not defined. Furthermore, a gauge transformation does not, in general, commute with taking time derivatives, that is the gauge transformation of a time derivative is not merely given by the time derivative of the gauge transformation.

Nevertheless, the gauge transformation of a time derivative can be computed with the help of

Lemma 1 For an arbitrary linear phase space function $\delta X$, the commutator between its gauge transformation and its time derivative is given (up to second order terms) by a single gauge transformation

$$
\delta_{\left[\xi_{0}, \xi\right]}(\delta \dot{X})-\left(\delta_{\left[\xi_{0}, \xi\right]} \delta X\right)^{\cdot}=\delta_{\left[0, \xi_{0}\right]} \delta X
$$

Proof: Using the definition of the gauge transformation (38) and time derivative (26) via the Poisson bracket, by virtue of the Jacobi identity we obtain

$$
\begin{aligned}
\delta_{\left[\xi_{0}, \xi\right]}(\delta \dot{X})-\left(\delta_{\left[\xi_{0}, \xi\right]} \delta X\right)^{\cdot}= & \left\{\left\{\delta X, H[N]+D\left[N^{a}\right]\right\}, H\left[\bar{N} \xi_{0}\right]+D\left[\partial^{a} \xi\right]\right\} \\
& \left.-\left\{\left\{\delta X, H\left[\bar{N} \xi_{0}\right]+D\left[\partial^{a} \xi\right]\right\}, H[N]+D\left[N^{a}\right]\right\}\right\} \\
= & \left\{\delta X,\left\{H[N]+D\left[N^{a}\right], H\left[\bar{N} \xi_{0}\right]+D\left[\partial^{a} \xi\right]\right\}\right\} .
\end{aligned}
$$

The inner Poisson bracket can be computed using the constraint algebra. In perturbative form, we have 2 ]

$$
\begin{aligned}
\left\{H[N]+D\left[N^{a}\right], H\left[\bar{N} \xi_{0}\right]+D\left[\partial^{a} \xi\right]\right\}= & D\left[\frac{1}{\bar{p}}\left(N \partial^{a}\left(\bar{N} \xi_{0}\right)-\bar{N} \xi_{0} \partial^{a} N\right)\right]-H\left[\partial^{a} \xi \partial_{a} N\right] \\
& +H\left[N^{a} \partial_{a}\left(\bar{N} \xi_{0}\right)\right]+D\left[\partial^{c} \xi \partial_{c} N^{a}-N^{c} \partial_{c} \partial^{a} \xi\right](48)
\end{aligned}
$$

Most of these constraint terms are at least of second order, and constraints whose Lagrange multiplier is quadratic will not affect the (leading) linear part of the gauge transformation of $\delta X$. Therefore the only relevant contribution comes from the first part of the first term, $D\left[\frac{\bar{N}}{\bar{p}} \partial^{a}\left(\bar{N} \xi_{0}\right)\right]$, which is equivalent to a single diffeomorphism transformation with the (linear part of the) shift vector given by

$$
\frac{\bar{N}}{\bar{p}} \partial^{a}\left(\bar{N} \xi_{0}\right)=\partial^{a} \xi_{0}+O(2) .
$$

The commutator then reads

$$
\delta_{\left[\xi_{0}, \xi\right]}(\delta \dot{X})-\left(\delta_{\left[\xi_{0}, \xi\right]} \delta X\right)^{\cdot}=\left\{\delta X, D\left[\partial^{a} \xi_{0}\right]\right\} \equiv \delta_{\left[0, \xi_{0}\right]} \delta X
$$

which is (46).

The last equation implies that diffeomorphism invariant canonical variables do have commuting time derivative and gauge transformation. Moreover, the leading diffeomorphism term originates from the Poisson bracket of the two Hamiltonian constraints on the left 
hand side of (48). Therefore, taking a time derivative commutes (up to quadratic terms) with a diffeomorphism transformation. This can also be seen from the absence of $\xi$ on the right hand side of (46). In other words, gauge transformations which do not involve the Hamiltonian constraint (i.e. such that $\xi_{0}=0$ ) commute with taking time derivatives.

For later convenience we write out gauge transformed time derivatives for a number of phase space variables:

$$
\begin{aligned}
\delta_{\left[\xi_{0}, \xi\right]}(\dot{\psi}) & =\left(\delta_{\left[\xi_{0}, \xi\right]} \psi\right)^{\cdot} \\
\delta_{\left[\xi_{0}, \xi\right]}(\dot{E}) & =\left(\delta_{\left[\xi_{0}, \xi\right]} E\right)^{\cdot}+\xi_{0} \\
\delta_{\left[\xi_{0}, \xi\right]}(\delta \dot{\varphi}) & =\left(\delta_{\left[\xi_{0}, \xi\right]} \delta \varphi\right)^{\cdot} \\
\delta_{\left[\xi_{0}, \xi\right]}(\delta \dot{\pi}) & =\left(\delta_{\left[\xi_{0}, \xi\right]} \delta \pi\right)^{\cdot}+\pi \Delta \xi_{0},
\end{aligned}
$$

where the gauge transformations of the triad components, following from (43) by comparison with (9), are given by

$$
\delta_{\left[\xi_{0}, \xi\right]} \psi=-\mathscr{H} \xi_{0} \quad, \quad \delta_{\left[\xi_{0}, \xi\right]} E=\xi .
$$

We now have all the ingredients to obtain the transformations of lapse and shift perturbations which (for the scalar mode and in conformal time) are expressed as in (10). Writing the perturbation of extrinsic curvature using the equation of motion

$$
\delta \dot{E}_{i}^{a} \equiv\left\{\delta E_{i}^{a}, H^{(2)}[\delta N]+D^{(2)}\left[\delta N^{a}\right]\right\}
$$

for the perturbed triad and the expression $\mathscr{H}=\dot{a} / a=\dot{\bar{p}} / 2 \bar{p}$ for the Hubble parameter, we obtain

$$
\delta K_{a}^{i}=-\delta_{a}^{i}[\dot{\psi}+\mathscr{H}(\psi+\phi)]+\partial_{a} \partial^{i}[\mathscr{H} E-(B-\dot{E})] .
$$

Performing a gauge transformation of the left hand side according to (43) and comparing to the gauge transformation of the right hand side using (49) and (50) yields the desired transformation of the Lagrange multipliers. Specifically, the diagonal part provides the transformed lapse perturbation

$$
\delta_{\left[\xi_{0}, \xi\right]} \phi=\dot{\xi}_{0}+\mathscr{H} \xi_{0},
$$

whereas the off-diagonal part implies

$$
\delta_{\left[\xi_{0}, \xi\right]}(B-\dot{E})=-\xi_{0} .
$$

Thus, the transformations of lapse and shift are indeed determined by gauge transformations of the phase space variables through the dynamical equations of motion.

Gauge invariant combinations are then obtained from the gauge transformations, which reproduces the Bardeen variables (35). Note that, as follows from (53), the quantity $B-\dot{E}$ is diffeomorphism invariant. According to Lemma 1, the gauge transformation of 
its time derivative is then given simply by the time derivative of its gauge transformation. Consequently, the last term in the $\Phi$-equation (35) gauge-transforms as

$$
\delta_{\left[\xi_{0}, \xi\right]}\{(B-\dot{E})\}=\left\{\delta_{\left[\xi_{0}, \xi\right]}(B-\dot{E})\right\}=-\dot{\xi}_{0},
$$

which along with the second term of $\Phi$ compensates the gauge-dependence of $\phi$.

We have determined gauge transformations of lapse and shift by making sure that the form of equations of motion for $E_{i}^{a}$ and $K_{a}^{i}$ is invariant. Here, we used the basic fact that changing the lapse function and shift vector leads to a different space-time metric decomposition and hence a different form of the triad and extrinsic curvature as well as their evolution. A different slicing of space-time also affects the matter variables, e.g. the definition of the field momentum. Gauge transformations of the matter variables will induce transformations of $N$ and $N^{a}$ through Hamilton's equations for $\varphi$ and $\pi$ as they did for gravitational phase space variables. We must therefore ensure that the transformations of the lapse and shift generated in this way be consistent with those obtained in (52) and (53). Taking the gauge transformation of the left hand side of the equation of motion

$$
\begin{aligned}
\delta \dot{\varphi} & \equiv\left\{\delta \varphi, H^{(2)}[N]+D^{(2)}\left[N^{a}\right]\right\}=\frac{\bar{N}}{\bar{p}^{3 / 2}}\left(\delta \pi-\bar{\pi} \frac{\left(\delta E_{i}^{a} \delta_{a}^{i}\right)}{2 \bar{p}}\right)+\frac{\delta N}{\bar{p}^{3 / 2}} \pi \\
& =\frac{\delta \pi}{\bar{p}}+\frac{\bar{\pi}}{\bar{p}}(3 \psi-\Delta E+\phi)
\end{aligned}
$$

according to (49) and (43), and comparing it with the transformation of the right hand side, yields the transformation of the lapse perturbation, $\delta_{\left[\xi_{0}, \xi\right]} \phi=\dot{\xi}_{0}+\mathscr{H} \xi_{0}$ which agrees with Eq. (52). Repeating the procedure for the momentum equation

$$
\begin{aligned}
\delta \dot{\pi} & \equiv\left\{\delta \pi, H^{(2)}[N]+D^{(2)}\left[N^{a}\right]\right\}=\bar{N}\left[\sqrt{\bar{p}} \Delta \delta \varphi-\bar{p}^{3 / 2}\left(V_{, \varphi \varphi} \delta \varphi+V_{, \varphi} \frac{\left(\delta E_{i}^{a} \delta_{a}^{i}\right)}{2 \bar{p}}\right)\right] \\
& =\bar{p} \Delta \delta \varphi-\bar{p}^{2}\left(V_{, \varphi \varphi} \delta \varphi-V_{, \varphi}(3 \psi-\Delta E+\phi)\right)+\bar{\pi} \Delta B
\end{aligned}
$$

results in $\delta_{\left[\xi_{0}, \xi\right]} B=\dot{\xi}$. The latter along with (49) and (150), implying

$$
\delta_{\left[\xi_{0}, \xi\right]} \dot{E}=\left(\delta_{\left[\xi_{0}, \xi\right]} E\right)^{\cdot}+\xi_{0}=\dot{\xi}+\xi_{0},
$$

reproduces the correct gauge transformation (53). Thus a fixed transformation of lapse and shift provides the correct gauge transformation of both gravity and matter phase space variables. This is a further consistency property ensured by the first class nature of constraints.

For matter fields, the gauge invariant density and scalar field perturbations are

$$
\delta \rho^{\mathrm{GI}}=\delta \rho+\dot{\bar{\rho}}_{\varphi}(B-\dot{E}) ; \quad \delta \varphi^{\mathrm{GI}}=\delta \varphi+\dot{\bar{\varphi}}(B-\dot{E}) .
$$

It is convenient for cosmological applications to introduce the gauge-invariant quantities:

$$
\mathcal{R}=\Psi+\mathscr{H}\left(\frac{\delta \varphi^{\mathrm{GI}}}{\dot{\bar{\varphi}}}\right)=\psi+\mathscr{H}\left(\frac{\delta \varphi}{\dot{\bar{\varphi}}}\right)
$$




$$
-\zeta=\Psi+\mathscr{H}\left(\frac{\delta \rho^{\mathrm{GI}}}{\dot{\bar{\rho}}_{\varphi}}\right)=\psi+\mathscr{H} \frac{\delta \rho}{\dot{\bar{\rho}}_{\varphi}}
$$

and

$$
\mathcal{R}_{2}=\Phi-\mathscr{H}\left(\frac{\delta \varphi^{\mathrm{GI}}}{\dot{\bar{\varphi}}}\right)-\left(\frac{\delta \varphi^{\mathrm{GI}}}{\dot{\bar{\varphi}}}\right)=\phi-\mathscr{H}\left(\frac{\delta \varphi}{\dot{\bar{\varphi}}}\right)-\left(\frac{\delta \varphi}{\dot{\bar{\varphi}}}\right)^{\cdot}
$$

The following points are worth noting regarding the above three gauge-invariant quantities: (i) $\mathcal{R}$ provides information about the nature of the long wavelength perturbations i.e. when the perturbations have left the Hubble radius. More precisely, $\dot{\mathcal{R}}$ vanishes in the long wavelength limit if the perturbations are adiabatic [42, 43]. (ii) $\zeta$ refers to the 3 -curvature perturbations on uniform density hypersurfaces. As $\mathcal{R}, \zeta$ is also conserved in the large scales and quantifies the large angular scale temperature anisotropies in the cosmic microwave background. In the slow-roll limit, the two gauge-invariant quantities are identical and either of them can be used to quantify the primordial perturbations. (iii) Unlike $\zeta$ and $\mathcal{R}$, the Bardeen potential $\Phi$ evolves in time from Hubble exit until the re-entry during matter/radiation era. More precisely, during inflation, at the super-Hubble scales,

$$
\Phi \simeq \epsilon_{s r} \zeta \quad \Longrightarrow \quad \Phi \simeq \epsilon_{s r}|A|
$$

where $\epsilon_{s r}$ is the slow-roll parameter which is much less than unity and $|A|$ is the value of $\zeta$ at the super-Hubble scales. However, at horizon re-entry, $\Phi \sim \zeta$. (iv) $\mathcal{R}$ (and also $\zeta$ ) is linearly related to the Mukhanov-Sasaki variable $Q$ which is useful for studying the quantization of perturbations. (v) Unlike $\mathcal{R}$ and $\zeta, \mathcal{R}_{2}$ is not often used in the study of cosmological perturbations. (vi) In the quantum corrected version of the perturbation equations, all the above quantities acquire non-trivial quantum corrections which we will discuss in the rest of the paper. This also affects the conservation of power at large scales.

For the classical gauge transformations we thus produce the well-known gauge invariant quantities (35), but we have now done so in a way which is entirely canonical. These methods therefore generalize directly to the case of equations and gauge transformations which are corrected by effects from quantum gravity even in quantum regimes where no underlying smooth space-time picture exists.

\subsection{Inclusion of quantum corrections}

With quantum gravity effects, both the equations of motion and gauge transformations are governed by the quantum corrected Hamiltonian constraint $H^{Q}$ in (14), including all the counter-terms and the diffeomorphism constraint (42) which remains unaffected. Recall also that the terms $\alpha^{\prime} p, \nu^{\prime} p$ as well as the counter-term functions are leading order quantum effects (not to be confused with perturbative order). From now on we neglect all higher order quantum corrections, such as $\left(\alpha^{\prime}\right)^{2} p^{2}, f^{2}, \alpha^{\prime} p f$ etc. Moreover, also in the background equations (30), (31) and (32) the quantum corrected terms must be used for consistency.

Gauge transformations are generated by the quantum corrected constraints with the lapse function and shift vector parameterized by the infinitesimal vector field $\xi^{\mu}=\left(\xi_{0}, \partial^{a} \xi\right)$ 
as

$$
\delta N=\bar{N} \xi_{0}, \quad \delta N^{a}=\partial^{a} \xi .
$$

The gauge transformation of the triad perturbation taking into account the counter-terms can be computed using the Poisson bracket

$$
\delta_{\left[\xi_{0}, \xi\right]} E_{i}^{a} \equiv\left\{\delta E_{i}^{a}, H^{Q(2)}\left[\bar{N} \xi_{0}\right]+D^{(2)}\left[\partial^{a} \xi\right]\right\}=2 \bar{\alpha} \bar{k} \bar{p} \xi_{0}(1+f) \delta_{i}^{a}+\bar{p}\left(\delta_{i}^{a} \Delta \xi-\partial^{a} \partial_{i} \xi\right)
$$

from which the transformations for $\psi$ and $E$ follow

$$
\delta_{\left[\xi_{0}, \xi\right]} \psi=-\mathscr{H} \xi_{0}(1+f), \quad \delta_{\left[\xi_{0}, \xi\right]} E=\xi
$$

The gauge transformed extrinsic curvature yields

$$
\delta_{\left[\xi_{0}, \xi\right]}\left(\alpha K_{a}^{i}\right)=\partial^{i} \partial_{a}\left(\mathscr{H} \xi+\bar{\alpha}^{2} \xi_{0}\right)-\left[\frac{1}{2} \mathscr{H}^{2} \xi_{0}(1+g)-4 \pi G \xi_{0} \bar{\alpha}\left(\bar{p} V(\bar{\varphi})-\frac{\dot{\bar{\varphi}}^{2}}{2 \bar{\nu}}\left(1+f_{2}\right)\right)\right] \delta_{a}^{i} .
$$

We compare this equation with the gauge transformation of (27), eliminating the potential term using the background Raychaudhuri equation (31). By virtue of the anomaly-freedom condition (22) along with [2]

$$
f_{2}=2 f_{1}
$$

the off-diagonal part results in

$$
\delta_{\left[\xi_{0}, \xi\right]}(B-\dot{E})=-\bar{\alpha}^{2} \xi_{0},
$$

whereas the diagonal part yields

$$
\delta_{\left[\xi_{0}, \xi\right]} \phi=\dot{\xi}_{0}+\mathscr{H} \xi_{0}\left(1+2 f^{\prime} \bar{p}+\frac{\bar{\alpha}^{\prime} \bar{p}}{\bar{\alpha}}\right) .
$$

Remarkably, the anomaly cancellation condition (20) implies that the last two terms inside the parenthesis mutually cancel, hence quantum corrected transformation of the lapse perturbation is equivalent to the classical one (52). Finally the matter perturbation transforms as

$$
\delta_{\left[\xi_{0}, \xi\right]} \delta \varphi=\dot{\bar{\varphi}}\left(1+f_{1}\right) \xi_{0} .
$$

The four metric perturbations can be combined into two gauge invariant quantum corrected potentials

$$
\begin{aligned}
\Psi & =\psi-\mathscr{H}(1+f) \frac{B-\dot{E}}{\bar{\alpha}^{2}} \\
\Phi & =\phi+\left(\frac{B-\dot{E}}{\bar{\alpha}^{2}}\right)+\mathscr{H} \frac{B-\dot{E}}{\bar{\alpha}^{2}} .
\end{aligned}
$$


Similarly, the gauge invariant matter variables are

$$
\delta \varphi^{\mathrm{GI}}=\delta \varphi+\dot{\bar{\varphi}}\left(1+f_{1}\right) \frac{B-\dot{E}}{\bar{\alpha}^{2}}
$$

Note that when omitting the quantum corrections in Eqs. (69) and (70) one recovers the classical results (35). From the corrected gauge invariant expressions, one can directly see that the combination

$$
\mathcal{R}=\psi+\frac{\mathscr{H}}{\dot{\bar{\varphi}}} \frac{1+f}{1+f_{1}} \delta \varphi=\Psi+\frac{\mathscr{H}}{\dot{\bar{\varphi}}} \frac{1+f}{1+f_{1}} \delta \varphi^{\mathrm{GI}},
$$

which does not refer to the non-trace perturbations $E$ and $B$, is gauge invariant. Also the explicit $\bar{\alpha}$-dependence drops out, showing that this particular perturbation is quantum corrected only because we were required to include counter-terms $f$ and $f_{1}$ in addition to the primary correction function $\bar{\alpha}$. Similarly the other curvature perturbation is

$$
\mathcal{R}_{2}=\phi-\mathscr{H} \frac{\delta \varphi}{\dot{\bar{\varphi}}} \frac{1}{1+f_{1}}-\left(\frac{\delta \varphi}{\dot{\bar{\varphi}}\left(1+f_{1}\right)}\right)=\Phi-\mathscr{H} \frac{\delta \varphi^{\mathrm{GI}}}{\dot{\bar{\varphi}}} \frac{1}{1+f_{1}}-\left(\frac{\delta \varphi^{\mathrm{GI}}}{\dot{\bar{\varphi}}\left(1+f_{1}\right)}\right)
$$

which does not refer to $\bar{\alpha}$, either. (A generalization of the perturbation $\zeta$ to quantum corrected equations requires a general derivation of $\delta \rho^{\mathrm{GI}}$, which will be done elsewhere.)

\section{Gauge invariant equations of motion}

We can now formulate the perturbed equations of motion purely in terms of the gauge invariant variables derived in the previous section. The following auxiliary relations will be useful:

$$
\begin{aligned}
\delta E_{i}^{a}= & -2 \bar{p} \Psi \delta_{i}^{a} \\
& -2 \mathscr{H} \bar{p}(1+f) \frac{B-\dot{E}}{\bar{\alpha}^{2}} \delta_{i}^{a}+\bar{p}\left(\delta_{i}^{a} \Delta-\partial^{a} \partial_{i}\right) E \\
\bar{\alpha} \delta K_{a}^{i}= & -\delta_{a}^{i}(\dot{\Psi}+\mathscr{H}(\Psi+\Phi(1+f))) \\
& -\delta_{a}^{i} \frac{B-\dot{E}}{\bar{\alpha}^{2}}\left(\dot{\mathscr{H}}(1+f)-\mathscr{H}^{2} \frac{\bar{\alpha}^{\prime} \bar{p}}{\bar{\alpha}}\right)+\partial_{a} \partial^{i}(\mathscr{H} E-(B-\dot{E}))
\end{aligned}
$$

where the first line of each equation contains only gauge invariant terms.

\subsection{Diffeomorphism constraint equation}

Varying the diffeomorphism constraint (4) with respect to the shift perturbation yields the diffeomorphism constraint equation (the space-time component of Einstein's equation):

$$
0=8 \pi G \alpha \frac{\delta D\left[N^{c}\right]}{\delta\left(\delta N^{c}\right)}=\bar{p}\left(\partial_{c}\left(\bar{\alpha} \delta K_{a}^{i} \delta_{i}^{a}\right)-\partial_{k}\left(\bar{\alpha} \delta K_{c}^{k}\right)\right)-\bar{\alpha} \bar{k} \partial_{d} \delta E_{k}^{d} \delta_{c}^{k}+8 \pi G \bar{\alpha} \bar{\pi} \partial_{c} \delta \varphi .
$$


Using the gauge invariant variables defined in (69) and (70), it can be rewritten as

$$
\partial_{c}\left[\dot{\Psi}+\mathscr{H}(1+f) \Phi-4 \pi G \frac{\bar{\alpha}}{\bar{\nu}} \dot{\varphi} \varphi^{\mathrm{GI}}\right]+(\text { gauge terms })=0,
$$

where the 'gauge terms' are

$$
2 \frac{B-\dot{E}}{\bar{\alpha}} \partial_{c}\left[-\dot{\mathscr{H}}(1+f)+\mathscr{H}^{2}\left(1+f+\frac{\bar{\alpha}^{\prime} \bar{p}}{\bar{\alpha}}\right)-4 \pi G \frac{\bar{\alpha}}{\bar{\nu}} \dot{\varphi}^{2}\left(1+f_{1}\right)\right]
$$

After eliminating the $\dot{\mathscr{C}}$-term using the background Raychaudhuri equation (31) the expression inside the square brackets of (76) becomes

$$
4 \pi G \frac{\bar{\alpha}}{\bar{\nu}} \dot{\bar{\varphi}}^{2}\left(f-f_{1}-\frac{\bar{\nu}^{\prime} \bar{p}}{3 \bar{\nu}}\right)
$$

which is proportional to one of the anomaly-freedom conditions (221). Thus all gauge dependent terms vanish and the diffeomorphism constraint equation takes the form

$$
\partial_{c}(\dot{\Psi}+\mathscr{H}(1+f) \Phi)=4 \pi G \frac{\bar{\alpha}}{\bar{\nu}} \dot{\varphi} \partial_{c} \delta \varphi^{\mathrm{GI}} .
$$

Note that classically the right hand side is nothing but the gauge invariant space-time component of the perturbed matter stress-energy tensor $-4 \pi G a^{2} \delta T_{S}^{T}$.

\subsection{Hamiltonian constraint equation}

As seen explicitly for the diffeomorphism constraint, for constraints which are part of a closed system, i.e. which result from an anomaly-free quantization, gauge invariance of the equations of motion is guaranteed and showing that the gauge dependent terms of a given equation do vanish is in general a rather tedious, although straightforward, exercise. We leave out such explicit demonstrations in this and the following sections.

The Hamiltonian constraint equation is obtained by variation with respect to the lapse perturbation:

$$
\begin{aligned}
\frac{\delta H^{Q}[N]}{\delta(\delta N)} & =\frac{1}{16 \pi G}\left[-4 \bar{\alpha} \bar{k} \sqrt{\bar{p}}(1+f) \delta K_{a}^{i} \delta_{i}^{a}-\frac{\bar{\alpha} \bar{k}^{2}}{\sqrt{\bar{p}}}(1+g) \delta E_{i}^{a} \delta_{a}^{i}+\frac{2 \bar{\alpha}}{\sqrt{\bar{p}}} \partial_{a} \partial^{i} \delta E_{a}^{i}\right] \\
& +\frac{\bar{\nu} \bar{\pi} \delta \pi}{\bar{p}^{3 / 2}}\left(1+f_{1}\right)-\left(\frac{\bar{\nu} \bar{\pi}^{2}}{2 \bar{p}^{3 / 2}}\left(1+f_{2}\right)-\bar{p}^{3 / 2} V(\varphi)\right) \frac{\delta E_{i}^{a} \delta_{a}^{i}}{2 \bar{p}}+\bar{p}^{3 / 2} V_{, \varphi}(\bar{\varphi})\left(1+f_{3}\right) \delta \varphi \\
& =0,
\end{aligned}
$$

where

$$
f_{3}=\frac{3}{2 \bar{p}^{3 / 2}} \int d \bar{p} \bar{p}^{1 / 2} f
$$

can be obtained from (142). 
Multiplying both sides by $\bar{\alpha} / \sqrt{\bar{p}}$ again allows one to replace the background extrinsic curvature with the Hubble rate. Then eliminating the field momentum and its perturbation using (29) and the auxiliary expressions (73) along with anomaly-freedom conditions of [2] to reduce the number of counter-term functions, one arrives at the gauge invariant Hamilton constraint equation (or perturbed Friedmann equation)

$$
\begin{aligned}
\Delta\left(\bar{\alpha}^{2} \Phi\right)-3 \mathscr{H}(1+f)[\dot{\Psi}+\mathscr{H} \Phi(1+f)]= & 4 \pi G \frac{\bar{\alpha}}{\bar{\nu}}\left(1+f_{3}\right)\left[\dot{\bar{\varphi}} \delta \dot{\varphi}^{\mathrm{GI}}-\dot{\bar{\varphi}}^{2}\left(1+f_{1}\right) \Phi\right. \\
& \left.+\bar{\nu} \bar{p} V_{, \varphi}(\bar{\varphi}) \delta \varphi^{\mathrm{GI}}\right]
\end{aligned}
$$

Again, the right hand side is nothing but the time-time component of the perturbed stressenergy tensor, which now includes quantum corrections.

\subsection{Hamilton's equations}

As mentioned before, each pair of Hamilton's equations for configuration variables and momenta can be combined into a single second order equation. We illustrate the procedure starting with the matter field. The time derivative of the momentum perturbation can be first computed using the Poisson bracket

$$
\begin{aligned}
\delta \dot{\pi}= & \left\{\delta \pi, H[N]+D\left[N^{a}\right]\right\}=\bar{\pi} \partial_{a} \delta N^{a}-\delta N \bar{p}^{3 / 2} V_{, \varphi}(\bar{\varphi})\left(1+f_{3}\right) \\
& +\bar{N}\left[\sqrt{\bar{p}} \bar{\sigma}\left(1+g_{5}\right) \Delta \delta \varphi-\bar{p}^{3 / 2} V_{, \varphi \varphi}(\bar{\varphi})\left(1+g_{6}\right) \delta \varphi-\bar{p}^{3 / 2} V_{, \varphi}(\bar{\varphi}) \frac{\delta E_{i}^{a} \delta_{a}^{i}}{2 \bar{p}}\right]
\end{aligned}
$$

and then compared to the time derivative of the right hand side of Eq. (29), which will include second time derivatives of the scalar field. With the help of the background equations and anomaly cancellations conditions, the Klein-Gordon equation can be cast in the gauge invariant form

$$
\begin{aligned}
\delta \ddot{\varphi}^{\mathrm{GI}} & +2 \mathscr{H} \delta \dot{\varphi}^{\mathrm{GI}}\left(1-\frac{\bar{\nu}^{\prime} \bar{p}}{\bar{\nu}}-g_{1}^{\prime} \bar{p}\right)-\bar{\nu} \bar{\sigma}\left(1-f_{3}\right) \Delta \delta \varphi^{\mathrm{GI}}+\bar{\nu} \bar{p} V,_{\varphi \varphi}(\bar{\varphi}) \delta \varphi^{\mathrm{GI}} \\
& +2 \bar{\nu} \bar{p} V_{, \varphi}(\bar{\varphi})\left(1+f_{1}\right) \Phi-\dot{\bar{\varphi}}\left[\left(1+f_{1}\right) \dot{\Phi}+3\left(1+g_{1}\right) \dot{\Psi}\right]-2 \mathscr{H} \dot{\bar{\varphi}}\left(f_{3}^{\prime} \bar{p}\right) \Phi=0 .
\end{aligned}
$$

Similarly one arrives at the spatial components of Einstein's equation. Taking the time derivative of Eq. (27) and noting that

$$
\left(\bar{\alpha} \delta K_{a}^{i}\right) \equiv \bar{\alpha} \delta \dot{K}_{a}^{i}+\delta K_{a}^{i} \dot{\bar{\alpha}}=\alpha \delta \dot{K}_{a}^{i}+2 \mathscr{H} \bar{\alpha} \delta K_{a}^{i}\left(\frac{\bar{\alpha}^{\prime} \bar{p}}{\bar{\alpha}}\right)
$$

one can substitute the time derivative of the extrinsic curvature perturbation using the Poisson bracket

$$
\delta \dot{K}_{a}^{i}=\left\{\delta K_{a}^{i}, H[N]+D\left[N^{b}\right]\right\} .
$$

The resulting expression will contain second order correction functions $\alpha^{(2)}$ and $\nu^{(2)}$ related to the background ones, $\bar{\alpha}$ and $\bar{\nu}$, by the conditions (23) for anomaly freedom. 
Taking the trace of each equation and substituting it back into the left hand side, one obtains

$$
\frac{\partial \alpha^{(2)}}{\partial\left(\delta E_{i}^{a}\right)}=\frac{\bar{\alpha}^{\prime} \delta E_{j}^{c}}{6 \bar{p}}\left(\delta_{c}^{j} \delta_{a}^{i}-2 \delta_{c}^{i} \delta_{a}^{j}\right), \quad \frac{\partial \nu^{(2)}}{\partial\left(\delta E_{i}^{a}\right)}=\frac{\bar{\nu}^{\prime} \delta E_{j}^{c}}{6 \bar{p}}\left(\delta_{c}^{j} \delta_{a}^{i}-2 \delta_{c}^{i} \delta_{a}^{j}\right)
$$

whose gauge invariant parts read

$$
\left(\frac{\partial \alpha^{(2)}}{\partial\left(\delta E_{i}^{a}\right)}\right)^{\mathrm{GI}}=-\frac{\bar{\alpha}^{\prime}}{3} \Psi \delta_{a}^{i}, \quad\left(\frac{\partial \nu^{(2)}}{\partial\left(\delta E_{i}^{a}\right)}\right)^{\mathrm{GI}}=-\frac{\bar{\nu}^{\prime}}{3} \Psi \delta_{a}^{i}
$$

The combined second order equation naturally decouples into two independent equations by taking diagonal and off-diagonal terms. After a tedious but rather straightforward computation, taking into account the background equations of motion together with the anomaly-freedom conditions, the former equation takes the form

$$
\begin{aligned}
\ddot{\Psi}+\mathscr{H}\left[2 \dot{\Psi}\left(1-\frac{\bar{\alpha}^{\prime} \bar{p}}{\bar{\alpha}}\right)+\dot{\Phi}(1+f)\right] & +\left[\dot{\mathscr{H}}+2 \mathscr{H}^{2}\left(1+f^{\prime} \bar{p}-\frac{\bar{\alpha}^{\prime} \bar{p}}{\bar{\alpha}}\right)\right] \Phi(1+f) \\
& =4 \pi G \frac{\bar{\alpha}}{\bar{\nu}}\left[\dot{\varphi} \delta \dot{\varphi}^{\mathrm{GI}}-\bar{p} \bar{\nu} V_{, \varphi}(\bar{\varphi}) \delta \varphi^{\mathrm{GI}}\right] .
\end{aligned}
$$

In the absence of anisotropic stress in the matter sector, which is the case for the scalar field, the gauge invariant part of the off-diagonal equation reads:

$$
\partial_{a} \partial^{i}\left(\bar{\alpha}^{2}(\Phi-\Psi(1+h))\right)=0
$$

which implies $\Phi=\Psi(1+h)$, replacing the classical relation $\Phi=\Psi$. Here, $h$ is a counterterm correction which has to satisfy

$$
h=-f+2 \frac{\bar{\alpha}^{\prime} \bar{p}}{\bar{\alpha}} .
$$

\section{Qualitative properties of the scalar perturbations}

In this section, we discuss salient properties of the scalar perturbations including inverse triad corrections from loop quantum gravity. For now, we do not compute the powerspectrum since this would involve reducing the perturbation equations (77), (80), (85), (82) into a single differential equation in terms of the Mukhanov-Sasaki variable. Instead, we will focus on aspects of the classical matter perturbations in an effective quantum spacetime. In particular, we show that (i) the speed of scalar perturbations is less than unity and can in fact be much smaller, and (ii) the scalar perturbations are not purely adiabatic and have a small entropic contribution arising from the quantum correction. 


\subsection{Speed of perturbations}

Using the relation $\Phi=(1+h) \Psi$, the perturbation equations (77), (80), (85) lead to

$$
\begin{aligned}
& \bar{\alpha}^{2}(1+h) \Delta \Psi-3 \mathscr{H}(1+f)[\dot{\Psi}+\mathscr{H}(1+f)(1+h) \Psi] \\
& =4 \pi G \frac{\bar{\alpha}}{\bar{\nu}}\left(1+f_{3}\right)\left[\dot{\bar{\varphi}} \delta \dot{\varphi}^{\mathrm{GI}}-\dot{\bar{\varphi}}^{2}\left(1+f_{1}\right) \Phi+\bar{\nu} \bar{p} V_{, \varphi}(\bar{\varphi}) \delta \varphi^{\mathrm{GI}}\right] \\
& \dot{\Psi}+\mathscr{H}(1+f)(1+h) \Psi=4 \pi G \frac{\bar{\alpha}}{\bar{\nu}} \dot{\varphi} \delta \varphi^{\mathrm{GI}} \\
& \ddot{\Psi}+\mathscr{H}\left[2\left(1-\frac{\bar{\alpha}^{\prime} \bar{p}}{\bar{\alpha}}\right) \dot{\Psi}+(1+f)(1+h) \dot{\Psi}\right] \\
& +\left[\dot{\mathscr{H}}+2 \mathscr{H}^{2}\left(1+f^{\prime} \bar{p}-\frac{\bar{\alpha}^{\prime} \bar{p}}{\bar{\alpha}}\right)+\frac{\dot{h}}{(1+h)}\right](1+f)(1+h) \Psi=4 \pi G \frac{\bar{\alpha}}{\bar{\nu}}\left[\dot{\varphi} \delta \dot{\varphi}^{\mathrm{GI}}-\bar{p} \bar{\nu} V_{, \varphi}(\bar{\varphi}) \delta \varphi^{\mathrm{GI}}\right] .
\end{aligned}
$$

Dividing (88) by $1+f_{3}$ and subtracting the result from Eq. (90) leads to

$$
\begin{aligned}
& \ddot{\Psi}-\bar{\alpha}^{2} \frac{1+h}{1+f_{3}} \Delta \Psi+\mathscr{H}\left[2\left(1-\frac{\bar{\alpha}^{\prime} \bar{p}}{\bar{\alpha}}\right)+(1+f)(1+h)+3 \frac{1+f}{1+f_{3}}\right] \dot{\Psi} \\
& {\left[\left\{\mathscr{H} \frac{h}{1+h}+\dot{\mathscr{H}}+2 \mathscr{H}^{2}\left(1+f^{\prime} \bar{p}-\frac{\bar{\alpha}^{\prime} \bar{p}}{\bar{\alpha}}\right)\right\}(1+f)(1+h)+\mathscr{H}^{2}(1+h)\right.} \\
& \left.\times\left\{3 \frac{(1+f)^{2}}{1+f_{3}}-\frac{1+\bar{\alpha}^{\prime} \bar{p} / \bar{\alpha}}{1-\bar{\nu}^{\prime} \bar{p} / \bar{\nu}}\left(1+f_{1}\right)\right\}+\dot{\mathscr{H}} \frac{\left(1+f_{1}\right)(1+h)}{1-\bar{\nu}^{\prime} \bar{p} / \bar{\nu}}\right] \Psi=-8 \pi G \bar{\alpha} \bar{p}\left(1+f_{3}\right) V_{, \bar{\varphi}} \delta \varphi^{\mathrm{GI}} .
\end{aligned}
$$

Replacing $V_{, \bar{\varphi}}$ and $\delta \varphi$ using the Klein-Gordon equation (32) and the diffeomorphism constraint (89), we obtain a second-order partial differential equation for $\Psi$ :

$$
\begin{aligned}
& \ddot{\Psi}-c_{s}^{2} \Delta \Psi+\left[2 \mathscr{H}\left\{1+\frac{3}{2} \frac{f-f_{3}}{1+f_{3}}+2 \frac{\bar{\nu}^{\prime}}{\bar{\nu}} \bar{p}+\frac{\bar{\alpha}^{\prime}}{\bar{\alpha}} \bar{p} f-\frac{f^{2}}{2}\right\}-2 \frac{\ddot{\bar{\varphi}}}{\dot{\bar{\varphi}}}\right] \dot{\Psi} \\
& {\left[\mathscr{H}\left\{\frac{\dot{h}}{1+h}-2 \frac{\ddot{\bar{\varphi}}}{\dot{\bar{\varphi}}}\right\}(1+f)+\dot{\mathscr{H}}\left\{\frac{1+f_{1}}{\left[1-\bar{\nu}^{\prime} \bar{p} /(3 \bar{\nu})\right]}+(1+f)\right\}+\mathscr{H}^{2}\left\{3 \frac{(1+f)^{2}}{1+f_{3}}\right.\right.} \\
& \left.\left.+2\left(1+f^{\prime} \bar{p}-\frac{\bar{\alpha}^{\prime} \bar{p}}{\bar{\alpha}}\right)(1+f)-\frac{\left(1+\bar{\alpha}^{\prime} \bar{p} / \bar{\alpha}\right)}{\left(1-\bar{\nu}^{\prime} \bar{p} /(3 \bar{\nu})\right)}\left(1+f_{1}\right)-4\left(1-\frac{\bar{\nu}^{\prime}}{\bar{\nu}} \bar{p}\right)(1+f)\right\}\right](1+h) \Psi=0
\end{aligned}
$$

where the second term shows the speed of perturbations

$$
c_{s}^{2}=\bar{\alpha}^{2} \frac{1+h}{1+f_{3}} .
$$

Eqs. (92) and (93) constitute one of the main results of this paper, which has the following implications:

(i) In the perturbative regime analyzed here, we have $\bar{\alpha}>1$ such that there is a danger of the speed of sound becoming super-luminal. However, as in the context of gravitational waves in [20], one must compare the propagation speed not with the classical 
speed of light (which is one) but with the physical speed of light of electromagnetic fields in the same effective quantum space-time. Since the Maxwell Hamiltonian is subject to quantum gravity corrections, too, [7, 44] the physical speed of light can differ from the classical one. In fact, for an anomaly-free coupling of the Maxwell field to gravity it must be larger than one by a factor which equals $\bar{\alpha}^{2}$ as shown in [20. The speed of perturbations derived here is super-luminal compared to the physical speed of light only if the remaining factor $(1+h) /\left(1+f_{3}\right)$ in (93) is larger than one. Using Eqs. (87) and (79) it is easy to show that, in the perturbative regime considered here (where $\bar{\alpha}^{\prime}$ is negative), we have $(1+h) /\left(1+f_{3}\right) \sim 1+h-f_{3}<1$. Thus, in the perturbative regime, the speed of scalar perturbations is indeed less than unity. Again, one can see the importance of consistency conditions for the counter-terms. (Although such a scenario arises in the case of non-canonical scalar field inflationary models [45], classically it is not possible for canonical scalar field inflation. Holonomy corrections, which have been used in [14] without ensuring consistency and anomaly-freedom, have been claimed to lead to a speed of sound much larger than unity and even divergent in some phases. This may indicate the inconsistency of the perturbation equations used there.)

(ii) From the corrected diffeomorphism constraint equation (89) one learns that in the absence of matter fields, the metric perturbation decays more slowly compared to the classical case. Assuming that $\bar{\alpha}$ is a slowly varying function, the metric perturbations decay as

$$
\Psi \propto \frac{1}{a^{(1+f)(1+h)}}
$$

According to (87), $f+h=2 \bar{\alpha}^{\prime} p / \bar{\alpha}<0$ (and $f h$ is subdominant compared to $f+h$ in the regime considered here). Hence, the decay should happen more slowly, implying that the inverse triad corrections enhance metric perturbations.

(iii) In the long wavelength limit, the second term in Eq. (92) can be neglected and the perturbations can be treated to be independent of the wave-number $|\mathbf{k}|$. Assuming that $\bar{\alpha}$ is a slowly varying function, the Bardeen potential is given by

$$
\Psi \propto \epsilon_{s r} a^{\frac{3}{3-2 n_{\alpha}}-\frac{1}{2}}
$$

where the constant of proportionality is determined by the choice of quantum state defined at the initial epoch of inflation [42]. Comparing this expression with the corresponding classical equation (60) suggests that the primordial perturbations have a different behavior compared to their classical counterpart. For $0<n_{\alpha}<3 / 2$, the quantum perturbations enhance the primordial perturbations compared to the classical one. For $n_{\alpha}>3 / 2$, the perturbations decay and will lead to tiny primordial perturbations. This suggests that quantifying the primordial perturbations can, in principle, constrain the value of $n_{\alpha}>3 / 2$. We will discuss the implications of this in a future publication. 
(iv) Note that in arriving at the above perturbation equation, we have not used the perturbed Klein-Gordon equation (82). To obtain the primordial power spectrum, we would need to consider the combined evolution of the scalar and the metric perturbations $\delta \varphi^{\mathrm{GI}}$ and $\Psi$.

\subsection{Isocurvature perturbations}

For canonical scalar fields in the classical theory, $\mathcal{R}$ is conserved on large scales implying that the perturbations are adiabatic. It can be shown [42] that

$$
\dot{\mathcal{R}}_{\text {class }}=\frac{\mathscr{H}}{4 \pi G \dot{\varphi}^{2}} \Delta \Psi \quad \stackrel{k \rightarrow 0}{\longrightarrow} 0 .
$$

Thus, on these scales $\mathcal{R}$ is conserved. However quantum effects modify this and lead to small entropic perturbations. Taking a time derivative of Eq. (57) and using the diffeomorphism constraint (89) leads to

$$
\begin{aligned}
\dot{\mathcal{R}}= & \dot{\Psi}+\frac{1+f}{1+f_{1}} \frac{\bar{\nu}}{\bar{\alpha}}\left[\ddot{\Psi}+\left(\mathscr{H}(1+f)(1+h)+\frac{\mathscr{\mathscr { H }}}{\mathscr{H}}-2 \frac{\ddot{\bar{\varphi}}}{\dot{\bar{\varphi}}}\right) \dot{\Psi}\right. \\
& \left.+2(1+f)(1+h)\left(\dot{\mathscr{H}}-\mathscr{H} \frac{\ddot{\bar{\varphi}}}{\dot{\bar{\varphi}}}\right) \Psi+\mathscr{H}((1+f)(1+h))^{\cdot} \Psi\right] \frac{\mathscr{H}}{4 \pi G \dot{\bar{\varphi}}^{2}} \\
& +\left(\frac{(1+f) \bar{\nu}}{\left(1+f_{1}\right) \bar{\alpha}}\right)^{\cdot}(\dot{\Psi}+\mathscr{H}(1+f)(1+h) \Psi) \frac{\mathscr{H}}{4 \pi G \dot{\bar{\varphi}}^{2}}
\end{aligned}
$$

Eqs. (92) and (32) in the long wavelength limit imply

$$
\dot{\mathcal{R}} \simeq \frac{1+f}{1+f_{1}} \frac{\bar{\nu}}{\bar{\alpha}}\left[\left(\frac{3 \bar{\alpha}^{\prime} \bar{p}}{\bar{\alpha}}-\frac{4 \bar{\nu}^{\prime} \bar{p}}{\bar{\nu}}-3 \frac{f-f_{3}}{1+f_{3}}\right) \dot{\Psi}-\dot{h}(1+f) \Psi\right] \frac{\mathscr{H}^{2}}{4 \pi G \dot{\bar{\varphi}}^{2}}
$$

from which we infer that $\mathcal{R}$ is not conserved on large scales. Thus, perturbations generated during a single scalar field epoch are no longer purely adiabatic - perturbations contain a small entropic contribution. Although such an effect has been seen earlier in a class of Lorentz violating models [46, our example here is the first to show that the primordial perturbations from inflation are not purely adiabatic and always contain a small entropic perturbation. (Note that anomaly-freedom of our equations [2] ensures that there are no violations of Lorentz symmetry, although the specific form of symmetries can be quantum corrected.)

\section{Discussion}

Effective constraints lead to quantum corrections in equations of motion as well as in gauge transformations. Applied to general relativity, it is not only the dynamics but also 
the underlying space-time structure and the notion of covariance which are affected by quantum corrections. In [2] it was shown that the full constraint algebra, i.e. the set of structure functions, of canonical quantum gravity changes when quantum corrections of a loop quantization are included. While no gauge freedom is destroyed in the anomalyfree quantization used, the algebra is not the classical hypersurface deformation algebra as originally derived by Dirac for classical general relativity. The quantum corrected equations used are generally covariant, but the symmetry type of the underlying covariance is quantum corrected. A determination of the full gauge algebra of quantum gravity would require going beyond the leading perturbative order, which is not available so far. But the results of [2] show that quantum corrections in the algebra must arise. In particular, this implies that terms in an effective action of canonical quantum gravity cannot be simply of higher curvature form. There must be additional effects such as non-local terms or non-commutative manifold structures.

In [2] as well as in this paper only the inverse triad type of quantum corrections due to the effects of [6, 7] is considered. There are additional quantum corrections, one due to the use of holonomies and a generic one due to quantum back-reaction of fluctuations, correlations and higher moments of a quantum state [47]. For these corrections no anomalyfree version for perturbative inhomogeneities has been found yet, which indicates that there are severe consistency restrictions especially for holonomy corrections. (Quantum back-reaction is generic, such that the existence of consistent deformations is guaranteed by the work on effective gravity e.g. in [48, 49].) Despite this incomplete status of quantum gravity corrections, the different structures of the three types of corrections shows that it is not possible to cancel corrections from one type, such as the inverse triad corrections used here, by corrections of the other types. Thus, corrections of the form discussed here must be present in any cosmological perturbation theory based on loop quantum gravity.

Given anomaly-freedom of the constraints, it is possible to construct gauge invariant variables and recast the equations of motion in an entirely gauge invariant manner. Here, this analysis was done for the perturbative constraints of [2], incorporating inverse triad corrections of loop quantum gravity in a way which, to leading orders, is anomaly-free. The final equations (777), (80), (82), (85)), and (86)):

$$
\begin{aligned}
\partial_{c}(\dot{\Psi}+\mathscr{H}(1+f) \Phi)= & 4 \pi G \frac{\bar{\alpha}}{\bar{\nu}} \dot{\varphi} \partial_{c} \delta \varphi^{\mathrm{GI}} \\
\Delta\left(\bar{\alpha}^{2} \Phi\right)-3 \mathscr{H}(1+f)[\dot{\Psi}+\mathscr{H} \Phi(1+f)]= & 4 \pi G \frac{\bar{\alpha}}{\bar{\nu}}\left(1+f_{3}\right)\left[\dot{\bar{\varphi}} \delta \dot{\varphi}^{\mathrm{GI}}-\dot{\bar{\varphi}}^{2}\left(1+f_{1}\right) \Phi\right. \\
& \left.+\bar{\nu} \bar{p} V_{, \varphi}(\bar{\varphi}) \delta \varphi^{\mathrm{GI}}\right] \\
\ddot{\Psi}+\mathscr{H}\left[2 \dot{\Psi}\left(1-\frac{\bar{\alpha}^{\prime} \bar{p}}{\bar{\alpha}}\right)+\dot{\Phi}(1+f)\right]+ & {\left[\dot{\mathscr{H}}+2 \mathscr{H}^{2}\left(1+f^{\prime} \bar{p}-\frac{\bar{\alpha}^{\prime} \bar{p}}{\bar{\alpha}}\right)\right] \Phi(1+f) } \\
= & 4 \pi G \frac{\bar{\alpha}}{\bar{\nu}}\left[\dot{\varphi} \delta \dot{\varphi}^{\mathrm{GI}}-\bar{p} \bar{\nu} V_{, \varphi}(\bar{\varphi}) \delta \varphi^{\mathrm{GI}}\right] \\
\partial_{a} \partial^{i}\left(\bar{\alpha}^{2}(\Phi-\Psi(1+h))\right)= & 0 \\
\delta \ddot{\varphi}^{\mathrm{GI}}+2 \mathscr{H} \delta \dot{\varphi}^{\mathrm{GI}}\left(1-\frac{\bar{\nu}^{\prime} \bar{p}}{\bar{\nu}}-g_{1}^{\prime} \bar{p}\right) & -\bar{\alpha}^{2}\left(1-f_{3}\right) \Delta \delta \varphi^{\mathrm{GI}}+\bar{\nu} \bar{p} V_{, \varphi \varphi}(\bar{\varphi}) \delta \varphi^{\mathrm{GI}}
\end{aligned}
$$




$$
+2 \bar{\nu} \bar{p} V_{,}(\bar{\varphi})\left(1+f_{1}\right) \Phi-\dot{\bar{\varphi}}\left[\left(1+f_{1}\right) \dot{\Phi}+3\left(1+g_{1}\right) \dot{\Psi}\right]-2 \mathscr{H} \dot{\bar{\varphi}}\left(f_{3}^{\prime} \bar{p}\right) \Phi=0
$$

collected here from the last two sections are manifestly gauge invariant and reproduce the classical perturbed Einstein's equation if one omits the quantum corrections. Besides gauge invariance, there is also a consistency issue which arises since, on general grounds, there are three unknown scalar functions subject to five equations. Moreover Eqs. (99) and (102) can be used to eliminate two of these functions in terms of just one, say $\Phi$, which should satisfy the three remaining equations.

Another perspective on closure of the equations of motion is given by considering that the Klein-Gordon equation (103) is not independent. In the covariant formalism, it results from the energy conservation equation for the matter field: $\nabla_{\mu} T^{\mu \nu}=0$, the counterpart of the Bianchi identity of the gravitational sector. The latter equation is automatically satisfied by construction of the Einstein tensor. For this reason, the Klein-Gordon equation can be expressed in terms of the other equations and their derivatives. In the canonical formulation such an argument, referring to the Bianchi identity, is not available, especially at the effective level, for it is a priori not clear what kind of action might correspond to the quantum corrected constraints. Nonetheless, one can use the form of the Bianchi identity as guidance to explicitly check the redundancy of the Klein-Gordon equation. In addition, the Hamiltonian constraint equation is indeed a constraint, which restricts initial data, rather than a dynamical equation as it does not contain second order time derivatives. If the constraint is also preserved dynamically it does not break the consistency of the equations of motion.

In the canonical setting, this is realized if the constraints are first class, which is the case in the situation at hand to the orders considered. In fact, closure of the constraint algebra guarantees both gauge invariance of the equations of motion derived here and their consistency. In one of the equations, specifically in the diffeomorphism constraint equation (75), we have explicitly shown that all gauge dependent terms mutually cancel. Similar straightforward but tedious calculations for the other equations can be performed, but for brevity we did not present them here. Given the closure of the constraint algebra, such an explicit demonstration of vanishing of the gauge terms becomes unnecessary, although it may still serve as an independent consistency check.

In the light of this, one can arrive at the final gauge invariant equations of motion using the following shortcut. After computing the time derivative for the corresponding conjugate momentum using the Poisson bracket, it is possible to keep only the gauge invariant parts of the variables, dropping all the gauge terms $\left(B, E\right.$ and $\left(\delta \varphi^{\mathrm{GI}}-\delta \varphi\right)$ in our case). Although setting $B=0=E$ in the metric would amount to the longitudinal gauge, this procedure is not equivalent to fixing the longitudinal gauge prior to deriving the equations of motion. By doing so, one would loose variational equations by off-diagonal metric components and thus control on the off-diagonal spatial Einstein equation. Without that equation, the relationship between $\Phi$ and $\Psi$ would remain undetermined and one could only 'borrow' the relation between $\Phi$ and $\Psi$ from the classical picture. The latter has proven to be incorrect at the effective level, as can be seen from Eq. (86). Also effects of counter-terms, required for anomaly-freedom, could not be seen in a gauge-fixed analysis, which in general makes 
such effective equations inconsistent. The same remarks apply to other gauge choices, such as uniform gauge.

Terms which arise from a complete treatment of all gauge properties, but which could not be seen in a gauge-fixed analysis, do have physical implications. As an example, we can derive an evolution equation for curvature perturbations by subtracting Eq. (80) divided by $1+f_{3}$ from Eq. (85) in such a way that matter perturbations are canceled for a stiff fluid presented by the free scalar in the case $V(\varphi)=0$ :

$$
\begin{aligned}
& \ddot{\Psi}-\frac{\Delta\left(\alpha^{2} \Phi\right)}{1+f_{3}}+\mathscr{H}\left(\left(2\left(1-\frac{\alpha^{\prime} p}{\alpha}\right)+3 \frac{1+f}{1+f_{3}}\right) \dot{\Psi}+(1+f) \dot{\Phi}\right) \\
& +\left(\dot{\mathscr{H}}^{2-\frac{\nu^{\prime} p}{3 \nu}} \frac{\mathscr{H}^{\prime} p}{1-\frac{\nu^{\prime} p}{3 \nu}}\left(2\left(1+f^{\prime} p-\frac{\alpha^{\prime} p}{\alpha}\right)+3 \frac{(1+f)^{2}}{1+f_{3}}-\frac{1+\frac{\alpha^{\prime} p}{\alpha}}{1-\frac{\nu^{\prime} p}{3 \nu}}\right)\right) \Phi=0 .
\end{aligned}
$$

This equation is similar to (92), but more special because it was derived assuming a stiff fluid. For long-wave length modes one can ignore the Laplacian of $\Phi$ and arrive at an ordinary differential equation in time for only $\Psi$ if we also use the relation $\Phi=\Psi(1+h)$. The classical equation would then be solved by a decaying function $\Psi(\eta)$ as well as a constant mode because the scale factor for the stiff fluid case satisfies the classical equation $\dot{\mathscr{H}}+2 \mathscr{H}^{2}=0$ which makes the coefficient of $\Phi$ in the evolution equation vanish. This conservation of power on large scales [43] can be demonstrated for any perfect fluid by eliminating stress-energy components from the gauge-invariant equations. It can also be shown to be a direct consequence of the classical conservation law.

With quantum corrections, however, the coefficient of $\Phi$ does not cancel exactly and the constant mode disappears. First, the background equation is now corrected to

$$
\dot{\mathscr{H}}=-2 \mathscr{H}^{2}\left(1-\frac{1}{2} \frac{(\bar{\alpha} \bar{\nu})^{\prime} \bar{p}}{\bar{\alpha} \bar{\nu}}\right)
$$

which follows from a combination of (30) and (31) in the case of a vanishing potential. With this, and using our perturbativity assumptions on the correction functions, the coefficient of $\Phi$ in (104) is

$$
\mathscr{H}^{2}\left(2 f^{\prime} \bar{p}+6 f-3 f_{3}-\frac{\bar{\alpha}^{\prime} \bar{p}}{\bar{\alpha}}+\frac{5}{3} \frac{\bar{\nu}^{\prime} \bar{p}}{\bar{\nu}}\right)
$$

which does not have to vanish even if the anomaly cancellation conditions are used. This confirms the conclusions of [8] which initially were based on a gauge-fixed treatment in longitudinal gauge. However, here the signs of the correction terms are different (for instance, $f^{\prime}<0$ while $f>0$ ) such that it depends on the regime whether power is enhanced or suppressed.

The gauge invariant equations of motion can now be used to describe evolution of the curvature perturbations, e.g. during cosmological inflation. The small quantum corrections accumulated during a sufficiently long inflationary phase may potentially lead to detectable imprints on the surface of last scattering and be observed in the Cosmic Microwave Background. 


\section{Acknowledgements}

This work was supported in part by NSF grants PHY0748336 and PHY0456913. MK was supported by NSF grant PHY0114375. SS wishes to thank Roy Maartens and Kevin Vandersloot for discussions. He is being supported by the Marie Curie Incoming International Grant IIF-2006-039205. GH thanks IGC, Penn State where a part of this work was completed. GH is partially supported by NSERC of Canada.

\section{Appendix}

In this appendix we present the determination of gauge invariant equations of motion, done in the main text in the presence of quantum corrections, for the classical canonical theory. Resulting equations can be seen to agree with [42].

\section{A Gauge invariant equations of motion}

As we have seen earlier, the background phase space variables as well as the background lapse and shift remain unchanged under the infinitesimal gauge transformations (up to the second order). Therefore the equations of motion governing them are also gauge invariant. In the perturbed context, both the canonical fields and the Lagrange multipliers gaugetransform in a non-trivial way, and equations of motion should be formulated for the gauge-invariant variables. Here, we systematically derive the background and perturbed canonical equations of motion of classical linearized gravity. We also discuss the equivalence of the canonical system of equations and Einstein's equation.

\section{A.1 Background equations}

Since the background shift vector is zero, the background diffeomorphism constraint is identically satisfied. Therefore background equations are generated only by the background Hamiltonian constraint

$$
H^{(0)}[\bar{N}]=V_{0} \bar{N}\left[-\frac{3 \sqrt{\bar{p}} \bar{k}^{2}}{8 \pi G}+\frac{\bar{\pi}^{2}}{2 \bar{p}^{3 / 2}}+\bar{p}^{3 / 2} V(\bar{\varphi})\right],
$$

where $\bar{k}$ and $\bar{p}$ are the background (diagonal) components of the extrinsic curvature and triad respectively, $\bar{\varphi}$ and $\bar{\pi}$ are the background matter field and its conjugate momentum. (The parameter $V_{0}=\int \mathrm{d}^{3} x$ is again the coordinate volume of space or of a compact region in which the perturbations are introduced.) The background lapse, $\bar{N}=a \equiv \sqrt{\bar{p}}$, corresponds to the conformal time.

The Hamiltonian (107) gives rise to the constraint equation

$$
0=\frac{\partial H^{(0)}[\bar{N}]}{\partial \bar{N}}=V_{0}\left[-\frac{3 \sqrt{\bar{p}} \bar{k}^{2}}{8 \pi G}+\frac{\bar{\pi}^{2}}{2 \bar{p}^{3 / 2}}+\bar{p}^{3 / 2} V(\bar{\varphi})\right]
$$


and two pairs of Hamilton's equations of motion

$$
\begin{aligned}
& \dot{\bar{k}}=\left\{\bar{k}, H^{(0)}[\bar{N}]\right\}=-\frac{\bar{N} \bar{k}^{2}}{2 \sqrt{\bar{p}}}+4 \pi G \bar{N}\left(-\frac{\bar{\pi}^{2}}{\bar{p}^{5 / 2}}+\sqrt{\bar{p}} V(\bar{\varphi})\right) \\
& \dot{\bar{p}}=\left\{\bar{p}, H^{(0)}[\bar{N}]\right\}=2 \bar{N} \sqrt{\bar{p}} \bar{k}
\end{aligned}
$$

for the gravitational variables and

$$
\begin{aligned}
& \dot{\bar{\varphi}}=\left\{\bar{\varphi}, H^{(0)}[\bar{N}]\right\}=\frac{\bar{N} \bar{\pi}}{\bar{p}^{3 / 2}} \\
& \dot{\bar{\pi}}=\left\{\bar{\pi}, H^{(0)}[\bar{N}]\right\}=-\bar{N} \bar{p}^{3 / 2} V_{, \varphi}(\bar{\varphi})
\end{aligned}
$$

for the matter field. Note that the lapse $\bar{N}=\sqrt{\bar{p}}$ should be fixed consistently after computing the Poisson brackets. Then, as follows from Eq. (110), the background extrinsic curvature is nothing but the conformal Hubble parameter

$$
\bar{k}=\frac{\dot{\bar{p}}}{2 \bar{p}} \equiv \frac{\dot{a}}{a}=: \mathscr{H},
$$

whereas the field momentum is given by

$$
\bar{\pi}=\bar{p} \dot{\bar{\varphi}}
$$

Using the two relations above in (108) yields the Friedmann equation

$$
\mathscr{H}^{2}=\frac{8 \pi G}{3}\left(\frac{\dot{\bar{\varphi}}^{2}}{2}+\bar{p} V(\bar{\varphi})\right),
$$

whose right hand side is proportional to the background matter energy density. The gravitational equation (109) should be recognized as the Raychaudhuri equation

$$
\dot{\mathscr{H}}=\mathscr{H}^{2}-4 \pi G \dot{\bar{\varphi}}^{2}
$$

written in conformal time. Finally, combining the matter Hamilton's equations into a single second order equation results in the Klein-Gordon equation

$$
\ddot{\bar{\varphi}}+2 \mathscr{H} \dot{\bar{\varphi}}+\bar{p} V_{, \varphi}(\bar{\varphi})=0 \text {. }
$$

The unusual factor of 2 (instead of the standard one, 3) in the second term is again due to the choice of conformal time.

\section{A.2 Perturbed equations}

Perturbed equations of motion are generated by (the second order part of) both Hamiltonian and diffeomorphism constraints. Using the background and perturbed lapse function, the gravitational Hamiltonian constraint can be written as

$$
H_{\text {grav }}^{(2)}[N]=\frac{1}{16 \pi G} \int \mathrm{d}^{3} x\left[\bar{N} \mathcal{H}^{(2)}+\delta N \mathcal{H}^{(1)}\right],
$$


with the first- and second-order parts of the Hamiltonian density given by (40) and

$$
\begin{aligned}
\mathcal{H}^{(2)}=\sqrt{\bar{p}} \delta K_{c}^{j} \delta K_{d}^{k} \delta_{k}^{c} \delta_{j}^{d}-\sqrt{\bar{p}}\left(\delta K_{c}^{j} \delta_{j}^{c}\right)^{2}-\frac{2 \bar{k}}{\sqrt{\bar{p}}} \delta E_{j}^{c} \delta K_{c}^{j} \\
-\frac{\bar{k}^{2}}{2 \bar{p}^{3 / 2}} \delta E_{j}^{c} \delta E_{k}^{d} \delta_{c}^{k} \delta_{d}^{j}+\frac{\bar{k}^{2}}{4 \bar{p}^{3 / 2}}\left(\delta E_{j}^{c} \delta_{c}^{j}\right)^{2}-\frac{\delta^{j k}}{2 \bar{p}^{3 / 2}}\left(\partial_{c} \delta E_{j}^{c}\right)\left(\partial_{d} \delta E_{k}^{d}\right),
\end{aligned}
$$

respectively. The second order part of the diffeomorphism constraint reads

$$
D_{\text {grav }}^{(2)}\left[N^{a}\right]=\frac{1}{8 \pi G} \int_{\Sigma} \mathrm{d}^{3} x \delta N^{c}\left[\bar{p} \partial_{c}\left(\delta_{k}^{d} \delta K_{d}^{k}\right)-\bar{p}\left(\partial_{k} \delta K_{c}^{k}\right)-\bar{k} \delta_{c}^{k}\left(\partial_{d} \delta E_{k}^{d}\right)\right] .
$$

In the matter sector, we similarly have the Hamiltonian constraint

$$
H_{\text {matter }}^{(2)}[N]=\int_{\Sigma} \mathrm{d}^{3} x\left[\bar{N}\left(\mathcal{H}_{\pi}^{(2)}+\mathcal{H}_{\nabla}^{(2)}+\mathcal{H}_{\varphi}^{(2)}\right)+\delta N\left(\mathcal{H}_{\pi}^{(1)}+\mathcal{H}_{\varphi}^{(1)}\right)\right]
$$

with the densities (41) and

$$
\begin{aligned}
\mathcal{H}_{\pi}^{(2)}= & \frac{1}{2} \frac{\delta \pi^{2}}{\bar{p}^{3 / 2}}-\frac{\bar{\pi} \delta \pi}{\bar{p}^{3 / 2}} \frac{\delta_{c}^{j} \delta E_{j}^{c}}{2 \bar{p}}+\frac{1}{2} \frac{\bar{\pi}^{2}}{\bar{p}^{3 / 2}}\left(\frac{\left(\delta_{c}^{j} \delta E_{j}^{c}\right)^{2}}{8 \bar{p}^{2}}+\frac{\delta_{c}^{k} \delta_{d}^{j} \delta E_{j}^{c} \delta E_{k}^{d}}{4 \bar{p}^{2}}\right), \\
\mathcal{H}_{\nabla}^{(2)}= & \frac{1}{2} \sqrt{\bar{p}} \delta^{a b} \partial_{a} \delta \varphi \partial_{b} \delta \varphi \\
\mathcal{H}_{\varphi}^{(2)}= & \frac{1}{2} \bar{p}^{3 / 2} V_{, \varphi \varphi}(\bar{\varphi}) \delta \varphi^{2}+\bar{p}^{3 / 2} V_{, \varphi}(\bar{\varphi}) \delta \varphi \frac{\delta_{c}^{j} \delta E_{j}^{c}}{2 \bar{p}} \\
& +\bar{p}^{3 / 2} V(\bar{\varphi})\left(\frac{\left(\delta_{c}^{j} \delta E_{j}^{c}\right)^{2}}{8 \bar{p}^{2}}-\frac{\delta_{c}^{k} \delta_{d}^{j} \delta E_{j}^{c} \delta E_{k}^{d}}{4 \bar{p}^{2}}\right),
\end{aligned}
$$

along with the diffeomorphism constraint

$$
D_{\text {matter }}^{(2)}\left[N^{a}\right]=\int_{\Sigma} \mathrm{d}^{3} x \delta N^{c} \bar{\pi} \partial_{c} \delta \varphi
$$

In the expressions above, the triad perturbation has the form (9), whereas the perturbations of lapse and shift are given by (10).

Below we formulate the perturbed equations of motion purely in terms of the gauge invariant variables. The equations, as before, are of two types:

(i) Constraint equations, i.e. the Hamiltonian and Diffeomorphism constraints and

(ii) Dynamical (Hamilton's) equations.

The latter are those for the matter variables (one second order equation) and for the gravitational variables (two independent second order equations: diagonal and off-diagonal). The following auxiliary relation will be useful for deriving gauge-invariant equations:

$$
\begin{aligned}
\delta E_{i}^{a}= & -2 \bar{p} \Psi \delta_{i}^{a} \\
& -2 \mathscr{H} \bar{p}(B-\dot{E}) \delta_{i}^{a}+\bar{p}\left(\delta_{i}^{a} \Delta-\partial^{a} \partial_{i}\right) E \\
\delta K_{a}^{i}= & -\delta_{a}^{i}[\dot{\Psi}+\mathscr{H}(\Psi+\Phi)] \\
& -\delta_{a}^{i} \dot{\mathscr{H}}(B-\dot{E})+\partial_{a} \partial^{i}[\mathscr{H} E-(B-\dot{E})],
\end{aligned}
$$


where the first line of each equation contains only gauge invariant terms.

\section{A.2.1 Diffeomorphism constraint equation}

Varying the smeared diffeomorphism constraint with respect to the shift perturbation yields the diffeomorphism constraint equation (the space-time Einstein equation):

$$
0=8 \pi G \frac{\delta D\left[\delta N^{c}\right]}{\delta\left(\delta N^{c}\right)}=\bar{p}\left(\partial_{c}\left(\delta K_{a}^{i} \delta_{i}^{a}\right)-\partial_{k}\left(\delta K_{c}^{k}\right)\right)-\bar{k} \partial_{d} \delta E_{k}^{d} \delta_{c}^{k}+8 \pi G \bar{\pi} \partial_{c} \delta \varphi .
$$

Using the gauge invariant variables defined in (135) and (56), this equation can be rewritten as

$$
\partial_{c}\left[\dot{\Psi}+\mathscr{H} \Phi-4 \pi G \dot{\bar{\varphi}} \delta \varphi^{\mathrm{GI}}\right]+(\text { gauge terms })=0,
$$

where the 'gauge terms' are

$$
2(B-\dot{E}) \partial_{c}\left[-\dot{\mathscr{H}}+\mathscr{H}^{2}-4 \pi G \dot{\bar{\varphi}}^{2}\right]
$$

The expression inside the square brackets is nothing but the background Raychaudhuri equation (116). Thus all gauge dependent terms vanish and the diffeomorphism constraint equation takes the form

$$
\partial_{c}[\dot{\Psi}+\mathscr{H} \Phi]=4 \pi G \dot{\bar{\varphi}} \partial_{c} \delta \varphi^{\mathrm{GI}}
$$

whose right hand side should be recognized as the gauge invariant space-time component of the perturbed matter stress-energy tensor $-4 \pi G a^{2} \delta T_{S}^{T}$.

\section{A.2.2 Hamiltonian constraint equation}

The Hamiltonian constraint equation is obtained by variation with respect to the lapse perturbation:

$$
\begin{aligned}
\frac{\delta H}{\delta(\delta N)}= & \frac{1}{16 \pi G}\left[-4 \bar{k} \sqrt{\bar{p}}\left(\delta K_{a}^{i} \delta_{i}^{a}\right)-\frac{\bar{k}^{2}}{\sqrt{\bar{p}}} \delta E_{i}^{a} \delta_{a}^{i}+\frac{2}{\sqrt{\bar{p}}} \partial_{a} \partial^{i} \delta E_{a}^{i}\right] \\
& +\frac{\bar{\pi} \delta \pi}{\bar{p}^{3 / 2}}-\left(\frac{\bar{\pi}^{2}}{2 \bar{p}^{3 / 2}}-\bar{p}^{3 / 2} V(\bar{\varphi})\right) \frac{\delta E_{i}^{a} \delta_{a}^{i}}{2 \bar{p}}+\bar{p}^{3 / 2} V_{, \varphi}(\bar{\varphi}) \delta \varphi \\
= & 0 .
\end{aligned}
$$

Dividing both sides by $\sqrt{\bar{p}}$ allows one to replace the background extrinsic curvature with the Hubble rate. Then eliminating the field momentum and its perturbation in terms of the time derivatives of the scalar field (see (54)) and using the auxiliary expressions (124), one arrives at the gauge invariant Hamilton constraint equation (perturbed Friedmann equation)

$$
\Delta \Phi-3 \mathscr{H}[\dot{\Psi}+\mathscr{H} \Phi]=4 \pi G\left[\dot{\bar{\varphi}} \delta \dot{\varphi}^{\mathrm{GI}}-\dot{\bar{\varphi}}^{2} \Phi+\bar{p} V_{, \varphi}(\bar{\varphi}) \delta \varphi^{\mathrm{GI}}\right]
$$

Again, the right hand side is nothing but the time-time component of the perturbed stressenergy tensor. 


\section{A.2.3 Hamilton's equations}

The perturbed dynamical matter equations are computed using the Poisson bracket, giving rise to (54) and (55). Expressing $\delta \pi$ from the first equation and substituting into the second one, with the help of the background equations, the Klein-Gordon equation can be cast in the gauge invariant form

$$
\delta \ddot{\varphi}^{\mathrm{GI}}+2 \mathscr{H} \delta \dot{\varphi}^{\mathrm{GI}}-\Delta \delta \varphi^{\mathrm{GI}}+\bar{p} V_{, \varphi \varphi}(\bar{\varphi}) \delta \varphi^{\mathrm{GI}}+2 \bar{p} V_{, \varphi}(\bar{\varphi}) \Phi-\dot{\bar{\varphi}}(\dot{\Phi}+3 \dot{\Psi})=0 .
$$

Similarly one can arrive at the spatial Einstein equations. The first order equations are given by

$$
\begin{aligned}
\delta \dot{K}_{a}^{i} & \equiv\left\{\delta K_{a}^{i}, H^{(2)}[N]+D^{(2)}\left[N^{a}\right]\right\} \\
& =\frac{\bar{N}}{\bar{p}^{3 / 2}}\left[-\bar{k} \bar{p} \delta K_{a}^{i}-\frac{\bar{k}^{2}}{2} \delta E_{k}^{d} \delta_{d}^{i} \delta_{a}^{k}+\frac{\bar{k}^{2}}{4} \delta E_{k}^{d} \delta_{d}^{k} \delta_{a}^{i}+\frac{\delta^{i k}}{2} \partial_{a} \partial_{d} \delta E_{k}^{d}\right] \\
& +\frac{\delta N \bar{k}^{2}}{2 \sqrt{\bar{p}}} \delta_{a}^{i}-\frac{1}{\sqrt{\bar{p}}} \partial_{a} \partial^{i} \delta N+8 \pi G \frac{\delta H_{m}^{(2)}[N]}{\delta\left(\delta E_{i}^{a}\right)} \\
\delta \dot{E}_{i}^{a} & \equiv\left\{\delta E_{i}^{a}, H^{(2)}[N]+D^{(2)}\left[N^{a}\right]\right\} \\
& =\frac{\bar{N}}{\sqrt{\bar{p}}}\left[\bar{p} \delta K_{c}^{j} \delta_{i}^{c} \delta_{j}^{a}-\bar{p}\left(\delta K_{c}^{j} \delta_{j}^{c}\right) \delta_{i}^{a}-\delta E_{i}^{a}\right]+2 \delta N \bar{k} \sqrt{\bar{p}} \delta_{i}^{a}+\bar{p}\left(\delta_{i}^{a} \partial_{c} \delta N^{c}-\partial_{i} \delta N^{a}\right)
\end{aligned}
$$

The combined second order equation naturally decouples into two independent equations: diagonal and off-diagonal. After a tedious but rather straightforward computation, taking into account the background equations of motion, the former equation takes the form

$$
\ddot{\Psi}+\mathscr{H}(2 \dot{\Psi}+\dot{\Phi})+\left(\dot{\mathscr{C}}+2 \mathscr{H}^{2}\right) \Phi=4 \pi G\left(\dot{\bar{\varphi}} \delta \dot{\varphi}^{\mathrm{GI}}-\bar{p} V_{, \varphi}(\varphi) \delta \varphi^{\mathrm{GI}}\right) .
$$

In the absence of anisotropic stress in the matter sector, which is the case for the scalar field, the gauge invariant part of the off-diagonal equation reads:

$$
\partial_{a} \partial^{i}[\Phi-\Psi]=0,
$$

which implies $\Phi=\Psi$ for the Bardeen potentials.

\section{B Anomaly Cancellation Conditions}

In this appendix, we summarize the set of anomaly cancellation conditions containing counter-term coefficients. These conditions, in turn, determine the coefficients in terms of primary quantum correction functions $\alpha, \nu$ and $\sigma$, and they impose restrictions on the primary corrections. We note from the expressions of quantum corrected Hamiltonian densities (17), (18) that there are three such functions $(f, g, h)$ in the gravitational sector, six $\left(f_{1}, f_{2}, g_{1}, g_{2}, g_{3}, g_{5}\right)$ in the kinetic sector and two $\left(f_{3}, g_{6}\right)$ in the potential sector of scalar 
matter. Thus for the system under consideration we have a total of eleven initially undetermined functions contained in the counter-terms. These free functions are constrained by anomaly cancellation.

Invariance of counter-terms under diffeomorphisms [2] led to four conditions

$$
g=-2 f, f_{2}=2 f_{1}, g_{2}=g_{1}, g_{3}=2 g_{2}=2 g_{1}
$$

among these coefficients. These equations trivially lead to the solutions for $g, f_{2}, g_{2}$ and $g_{3}$, leaving seven functions to be determined. Cancellation of anomaly terms from the Poisson bracket between Hamiltonian constraints led to two independent conditions $\left(\mathcal{G}_{1}^{\varphi}=0, \mathcal{G}_{2}^{\varphi}=0\right.$ of [2]) from the gravitational sector. These two conditions along with the equation (135) imply

$$
\begin{aligned}
h & =-f+2 \frac{\bar{\alpha}^{\prime} \bar{p}}{\bar{\alpha}}, \\
2 \bar{p} f^{\prime} & =-\frac{\bar{\alpha}^{\prime} \bar{p}}{\bar{\alpha}} .
\end{aligned}
$$

These two equations explicitly solve $f$ and $h$ in terms of the primary correction function $\alpha$. In particular, for the given form of $\bar{\alpha}=1+c_{\alpha}\left(\ell_{\mathrm{P}}^{2} / \bar{p}\right)^{n_{\alpha}}+\cdots$, (137) has the solution

$$
f=\frac{1}{2 n_{\alpha}} \frac{\bar{\alpha}^{\prime} \bar{p}}{\bar{\alpha}}
$$

Thus, there are only five remaining functions that need to be determined. Three of the matter anomaly cancellation conditions $\left(\mathcal{D}_{1}=0, \mathcal{D}_{2}=0, \mathcal{B}_{4}^{\varphi}=0\right.$ of [2]) lead to

$$
g_{5}=-f_{1}, g_{6}=-g_{1}, f_{3}=f_{1}-g_{1}
$$

whereas another two conditions $\left(\mathcal{B}_{1}^{\varphi}=0, \mathcal{B}_{2}^{\varphi}=0\right.$ of [2]) solve $f_{1}$ and $g_{1}$ explicitly as

$$
\begin{aligned}
& f_{1}=f-\frac{\bar{\nu}^{\prime} \bar{p}}{3 \bar{\nu}}, \\
& g_{1}=\frac{\bar{\alpha}^{\prime} \bar{p}}{3 \bar{\alpha}}-\frac{\bar{\nu}^{\prime} \bar{p}}{\bar{\nu}}+\frac{2}{9}\left(\frac{\bar{\nu}^{\prime} \bar{p}}{\bar{\nu}}\right)^{\prime} p .
\end{aligned}
$$

At this stage all counter-terms coefficients have been determined. There is one remaining anomaly cancellation condition from the matter sector, $\mathcal{D}_{4}=0$ of [2], which implies

$$
2 \bar{p} f_{3}^{\prime}+3 f_{3}-3 f=0
$$

and thus requires the primary correction functions to satisfy

$$
\frac{\bar{\alpha}^{\prime} \bar{p}}{\bar{\alpha}}+\frac{\bar{p}}{3}\left(\frac{\bar{\alpha}^{\prime} \bar{p}}{\bar{\alpha}}\right)^{\prime}-\frac{\bar{\nu}^{\prime} \bar{p}}{\bar{\nu}}-\frac{\bar{p}}{9}\left(\frac{\bar{\nu}^{\prime} \bar{p}}{\bar{\nu}}\right)^{\prime}+\frac{2 \bar{p}^{2}}{9}\left(\frac{\bar{\nu}^{\prime} \bar{p}}{\bar{\nu}}\right)^{\prime \prime}=0 .
$$

Independent of the counter-terms and the the requirement (143), we also have the relation $\bar{\alpha}^{2}=\bar{\nu} \bar{\sigma}$ to be satisfied by the primary correction functions. Thus, anomaly freedom of the constraint algebra severely restricts the allowed form of primary quantum corrections functions, but it does permit non-trivial forms of quantum corrections. 


\section{References}

[1] M. Bojowald, Loop Quantum Cosmology, Living Rev. Relativity 11 (2008) 4, gr-qc/0601085, http://www . livingreviews .org/lrr-2008-4

[2] M. Bojowald, G. Hossain, M. Kagan, and S. Shankaranarayanan, Anomaly freedom in perturbative loop quantum gravity, Phys. Rev. D 78 (2008) 063547, arXiv:0806.3929

[3] C. Rovelli, Quantum Gravity, Cambridge University Press, Cambridge, UK, 2004

[4] A. Ashtekar and J. Lewandowski, Background independent quantum gravity: A status report, Class. Quantum Grav. 21 (2004) R53-R152, gr-qc/0404018

[5] T. Thiemann, Introduction to Modern Canonical Quantum General Relativity, Cambridge University Press, Cambridge, UK, 2007, gr-qc/0110034

[6] T. Thiemann, Quantum Spin Dynamics (QSD), Class. Quantum Grav. 15 (1998) 839-873, |gr-qc/9606089

[7] T. Thiemann, QSD V: Quantum Gravity as the Natural Regulator of Matter Quantum Field Theories, Class. Quantum Grav. 15 (1998) 1281-1314, [gr-qc/9705019]

[8] M. Bojowald, H. Hernández, M. Kagan, P. Singh, and A. Skirzewski, Formation and evolution of structure in loop cosmology, Phys. Rev. Lett. 98 (2007) 031301, astro-ph/0611685

[9] M. Bojowald, H. Hernández, M. Kagan, P. Singh, and A. Skirzewski, Hamiltonian cosmological perturbation theory with loop quantum gravity corrections, Phys. Rev. D 74 (2006) 123512, [gr-qc/0609057]

[10] D. J. Mulryne and N. J. Nunes, Constraints on a scale invariant power spectrum from superinflation in LQC, Phys. Rev. D 74 (2006) 083507, astro-ph/0607037]

[11] G. Calcagni and M. V. Cortês, Inflationary scalar spectrum in loop quantum cosmology, Class. Quantum Grav. 24 (2007) 829-853, gr-qc/0607059

[12] E. J. Copeland, D. J. Mulryne, N. J. Nunes, and M. Shaeri, Super-inflation in Loop Quantum Cosmology, Phys. Rev. D 77 (2008) 023510, arXiv:0708.1261

[13] M. SzydLowski, W. GodLowski, and T. Stachowiak, Cosmography in testing loop quantum gravity, Phys. Rev. D 77 (2008) 043530, arXiv:0706.0283.

[14] M. Artymowski, Z. Lalak, and L. Szulc, Loop Quantum Cosmology corrections to inflationary models, arXiv:0807.0160

[15] D. S. Salopek and J. R. Bond, Nonlinear evolution of long wavelength metric fluctuations in inflationary models, Phys. Rev. D 42 (1990) 3936-3962 
[16] D. Wands, K. A. Malik, D. H. Lyth, and A. R. Liddle, A new approach to the evolution of cosmological perturbations on large scales, Phys. Rev. D 62 (2000) 043527, astro-ph/0003278

[17] E. Bertschinger, On the Growth of Perturbations as a Test of Dark Energy, Astrophys. J. 648 (2006) 797, astro-ph/0604485

[18] P. A. M. Dirac, Lectures on Quantum Mechanics, Yeshiva Press, 1969

[19] M. Bojowald and G. Hossain, Cosmological vector modes and quantum gravity effects, Class. Quantum Grav. 24 (2007) 4801-4816, arXiv:0709.0872

[20] M. Bojowald and G. Hossain, Quantum gravity corrections to gravitational wave dispersion, Phys. Rev. D 77 (2008) 023508, arXiv:0709.2365

[21] C. Rovelli and L. Smolin, Loop Space Representation of Quantum General Relativity, Nucl. Phys. B 331 (1990) 80-152

[22] C. Rovelli and L. Smolin, Discreteness of Area and Volume in Quantum Gravity, Nucl. Phys. B 442 (1995) 593-619, [gr-qc/9411005], Erratum: Nucl. Phys. B 456 (1995) 753

[23] A. Ashtekar and J. Lewandowski, Quantum Theory of Geometry I: Area Operators, Class. Quantum Grav. 14 (1997) A55-A82, gr-qc/9602046

[24] A. Ashtekar and J. Lewandowski, Quantum Theory of Geometry II: Volume Operators, Adv. Theor. Math. Phys. 1 (1997) 388-429, gr-qc/9711031]

[25] C. Rovelli and L. Smolin, The physical Hamiltonian in nonperturbative quantum gravity, Phys. Rev. Lett. 72 (1994) 446-449, gr-qc/9308002

[26] M. Bojowald, H. Hernández, M. Kagan, and A. Skirzewski, Effective constraints of loop quantum gravity, Phys. Rev. D 75 (2007) 064022, [gr-qc/0611112]

[27] A. Ashtekar, New Hamiltonian Formulation of General Relativity, Phys. Rev. D 36 (1987) 1587-1602

[28] J. F. Barbero G., Real Ashtekar Variables for Lorentzian Signature Space-Times, Phys. Rev. D 51 (1995) 5507-5510, gr-qc/9410014]

[29] G. Immirzi, Real and Complex Connections for Canonical Gravity, Class. Quantum Grav. 14 (1997) L177-L181

[30] M. Bojowald, Inverse Scale Factor in Isotropic Quantum Geometry, Phys. Rev. D 64 (2001) 084018, gr-qc/0105067]

[31] M. Bojowald, Quantization ambiguities in isotropic quantum geometry, Class. Quantum Grav. 19 (2002) 5113-5130, gr-qc/0206053] 
[32] M. Bojowald, Loop Quantum Cosmology: Recent Progress, Pramana 63 (2004) 765776, In Proceedings of the International Conference on Gravitation and Cosmology (ICGC 2004), Cochin, India, gr-qc/0402053

[33] J. Brunnemann and T. Thiemann, Unboundedness of Triad-Like Operators in Loop Quantum Gravity, Class. Quantum Grav. 23 (2006) 1429-1483, grr-qc/0505033]

[34] M. Bojowald, Loop quantum cosmology and inhomogeneities, Gen. Rel. Grav. 38 (2006) 1771-1795, gr-qc/0609034]

[35] M. Bojowald, The dark side of a patchwork universe, Gen. Rel. Grav. 40 (2008) 639-660, arXiv:0705.4398]

[36] G. Calcagni and G. Hossain, Loop quantum cosmology and tensor perturbations in the early universe, Adv. Sci. Lett. (2008) to appear, arXiv:0810.4330]

[37] B. Dittrich and J. Tambornino, A perturbative approach to Dirac observables and their space-time algebra, Class. Quant. Grav. 24 (2007) 757-784, [gr-qc/0610060]

[38] B. Dittrich and J. Tambornino, Gauge invariant perturbations around symmetry reduced sectors of general relativity: applications to cosmology, Class. Quantum Grav. 24 (2007) 4543-4585, [gr-qc/0702093]

[39] K. Giesel, S. Hofmann, T. Thiemann, and O. Winkler, Manifestly Gauge-Invariant General Relativistic Perturbation Theory: II. FRW Background and First Order, arXiv:0711.0117]

[40] J. M. Bardeen, Gauge-invariant cosmological perturbations, Phys. Rev. D 22 (1980) 1882-1905

[41] J. M. Pons, D. C. Salisbury, and L. C. Shepley, Gauge transformations in the Lagrangian and Hamiltonian formalisms of generally covariant theories, Phys. Rev. D 55 (1997) 658-668, gr-qc/9612037

[42] V. F. Mukhanov, H. A. Feldman, and R. H. Brandenberger, Theory of cosmological perturbations, Phys. Rept. 215 (1992) 203-333

[43] D. H. Lyth, Large Scale Energy Density Perturbations And Inflation, Phys. Rev. D $31(1985) 1792$

[44] M. Bojowald and R. Das, The radiation equation of state and loop quantum gravity corrections, Phys. Rev. D 75 (2007) 123521, arXiv:0710.5721]

[45] J. Garriga and V. F. Mukhanov, Perturbations in k-inflation, Phys. Lett. B 458 (1999) 219, hep-th/9904176

[46] S. Shankaranarayanan and M. Lubo, Gauge-invariant perturbation theory for transPlanckian inflation, Phys. Rev. D 72 (2005) 123513, hep-th/0507086 
[47] M. Bojowald and A. Skirzewski, Quantum Gravity and Higher Curvature Actions, Int. J. Geom. Meth. Mod. Phys. 4 (2007) 25-52, [hep-th/0606232], Proceedings of "Current Mathematical Topics in Gravitation and Cosmology" (42nd Karpacz Winter School of Theoretical Physics), Ed. Borowiec, A. and Francaviglia, M.

[48] J. F. Donoghue, Leading Quantum Correction to the Newtonian Potential, Phys. Rev. Lett. 72 (1994) 2996-2999, gr-qc/9310024

[49] J. F. Donoghue, General relativity as an effective field theory: The leading quantum corrections, Phys. Rev. D 50 (1994) 3874-3888, gr-qc/9405057] 